\title{
More on R-Union and R-Intersection of Neutrosophic Soft Cubic Set
}

\section{R. ANITHA CRUZ ${ }^{1}$ AND F. NIRMALA IRUDAYAM ${ }^{2}$}

${ }^{1} \mathrm{R}$. Anitha Cruz, Department of Mathematics Nirmala College for Women, Coimbatore, 64101893, India,

${ }^{2} \mathrm{~F}$. Nirmala Irudayam, Assistant Professor, Department of Mathematics, Nirmala

College for Women,Coimbatore, 641018, India, nirmalairudayam@ymail.com

Email: anithacruz@gmail.com

Received: February 4, 2018I Revised: February 16,2018I Accepted: February 21, 2018

Published online: March 01, 2018

The Author(s) 2018. This article is published with open access at www.chitkara.edu.in/publications

Abstract R-unions and R-intersections, R-OR, R-AND of Neutrosophic soft cubic sets are introduced and related properties are investigated. We show that the R-union (R-intersection) of internal neutrosophic soft cubic set is also an internal neutrosophic soft cubic set. We show that the R-union and the R-intersection T-external (I-external, F-external) neutrosophic soft cubic sets are also T-external ( I-external, F-external) neutrosophic soft cubic sets. The conditions for the R-intersection of two cubic soft sets to be both an external neutrosophic soft cubic set and an internal neutrosophic soft cubic set. Further we provide a condition for the R- intersection and $\mathrm{R}$ union of two $\mathrm{T}$-internal (I-internal, F-internal) neutrosophic soft cubic sets are T-external (I-external, F-external) neutrosophic soft cubic sets.

Keywords: Neutrosophic soft cubic set, T-internal (resp. I- internal, F-internal) neutrosophic soft cubic sets, T-external (resp. I- external, F-external)

neutrosophic soft cubic set, R-union, R-intersection of neutrosophic soft cubic set.

\section{INTRODUCTION}

Every real situation does not have a crisp or an exact solution hence there is some degree of uncertainty. To deal with uncertainty many Mathematician developed many theories. In 1965 Zadeh [19] introduced the concept of Fuzzy set were we consider the degree of belongingness to a set as a membership function. Following him in 1986 Atanassov [3] introduced the degree of non membership and defined intuitionistic fuzzy set. Further researches were done in these fields but these two sets were not enough to meet all the uncertainties in

\footnotetext{
Mathematical Journal of Interdisciplinary Sciences Vol-6, No-2, March 2018 pp. 93-117
} 
Cruz, RA

Irudayam, FN

real physical problems. Hence In 1995 Smarandache [5, 6] coined neutrosophic logic and neutrosophic sets to deal with truth, indeterminate and falsehood. On other hand in 1999 Molodtsov [4] introduced soft set which helps the view an environment in a parameterized manner. Pabita Kumar Majii [5-7] had combined the Neutrosophic set with soft sets and introduced 'Neutrosophic soft set'. Y. B. Jun et al. [16-18] coined cubic set by using a fuzzy set and an interval-valued fuzzy set, and also extended the concept of cubic sets to the neutrosophic cubic sets. . [1] Introduced neutrosophic soft cubic set and the notion of truth-internal (indeterminacy-internal, falsity-internal) neutrosophic soft cubic sets and truth-external (indeterminacy-internal, falsity-internal) neutrosophic soft cubic sets.

As a continuation of the paper [1] we consider R-unions and R-intersections of T-external (I-external, F-external) neutrosophic soft cubic sets. We provide examples to show that the R-intersection and the R-union of T-external (resp. I-external and F-external) neutrosophic soft cubic sets may not be a T-external (resp. I-external and F-external) neutrosophic soft cubic set. We also discuss conditions for the R-union of T-external (resp. I-external and F-external) neutrosophic soft cubic sets to be a T-external (resp. I-external and F-external) neutrosophic soft cubic set. Further the condition for the R-intersection of T-external (resp. I-external and F-external) neutrosophic soft cubic sets to be a T-external (resp. I-external and F-external) neutrosophic soft cubic set.

\section{PRELIMINARIES}

2.1 Definition [19] Let $E$ be a universe. Then a fuzzy set $\mu$ over $E$ is defined by $X=\left\{\mu_{x}(x) / x: x \in E\right\}$ where $\mu_{x}$ is called membership function of $X$ and defined by $\mu_{\mathrm{x}}: \mathrm{E} \rightarrow[0,1]$. For each $\mathrm{x} E$, the value $\mu_{\mathrm{x}}(\mathrm{x})$ represents the degree of $\mathrm{x}$ belonging to the fuzzy set $\mathrm{X}$.

2.2 Definition: [16] Let $\mathrm{X}$ be a non-empty set. By a cubic set, we mean a structure $\Xi=\{\langle x, A(x), \mu(x)\rangle \mid x \in X\}$ in which $\mathrm{A}$ is an interval valued fuzzy set (IVF) and $\mu$ is a fuzzy set. It is denoted by $\langle A, \mu\rangle$.

2.3 Definition: [5] Let $U$ be an initial universe set and $E$ be a set of parameters. Consider $\mathrm{A} \subset \mathrm{E}$. Let $\mathrm{P}(\mathrm{U})$ denotes the set of all neutrosophic sets of $\mathrm{U}$. The collection $(F, A)$ is termed to be the soft neutrosophic set over $U$, where $F$ is a mapping given by $\mathrm{F}: \mathrm{A} \rightarrow \mathrm{P}(\mathrm{U})$.

2.4 Definition: [9] Let $X$ be an universe. Then a neutrosophic (NS) set $\lambda$ is an object having the form $\lambda=\{<\mathrm{x}: \mathrm{T}(\mathrm{x}), \mathrm{I}(\mathrm{x}), \mathrm{F}(\mathrm{x})\rangle: \mathrm{x} \in \mathrm{X}\}$ where the functions $\mathrm{T}, \mathrm{I}, \mathrm{F}: \mathrm{X} \rightarrow]^{-0} 0,1+[$ defines respectively the degree of Truth, the degree of indeterminacy, and the degree of falsehood of the element $\mathrm{x} \in \mathrm{X}$ to the set $\lambda$ with the condition. 


$$
-0 \leq \mathrm{T}(\mathrm{x})+\mathrm{I}(\mathrm{x})+\mathrm{F}(\mathrm{x}) \leq 3^{+}
$$

2.5 Definition: [15] Let $X$ be a non-empty set. An interval neutrosophic set (INS) $\mathrm{A}$ in $\mathrm{X}$ is characterized by the truth-membership function $\mathrm{A}_{\mathrm{T}}$, the indeterminacy-membership function $\mathrm{A}_{\mathrm{I}}$ and the falsity-membership function $\mathrm{A}_{\mathrm{F}}$. For each point $\mathrm{x} \in \mathrm{X}, \mathrm{A}_{\mathrm{T}}(\mathrm{x}), \mathrm{A}_{\mathrm{I}}(\mathrm{x}), \mathrm{A}_{\mathrm{F}}(\mathrm{x}) \subseteq[0,1]$.

For two INS

$\mathrm{A}=\left\{<\mathrm{x},\left[\mathrm{A}_{\mathrm{T}}^{-}(\mathrm{x}), \mathrm{A}_{\mathrm{T}}^{+}(\mathrm{x})\right],\left[\mathrm{A}_{\mathrm{I}}^{-}(\mathrm{x}), \mathrm{A}_{\mathrm{I}}^{+}(\mathrm{x})\right],\left[\mathrm{A}_{\mathrm{F}}^{-}(\mathrm{x}), \mathrm{A}_{\mathrm{F}}^{+}(\mathrm{x})\right]>: \mathrm{x} \in \mathrm{X}\right\}$

And

$\mathrm{B}=\left\{<\mathrm{x},\left[\mathrm{B}_{\mathrm{T}}^{-}(\mathrm{x}), \mathrm{B}_{\mathrm{T}}^{+}(\mathrm{x})\right],\left[\mathrm{B}_{\mathrm{I}}^{-}(\mathrm{x}), \mathrm{B}_{\mathrm{I}}^{+}(\mathrm{x})\right],\left[\mathrm{B}_{\mathrm{F}}^{-}(\mathrm{x}), \mathrm{B}_{\mathrm{F}}^{+}(\mathrm{x})\right]>: \mathrm{x} \in \mathrm{X}\right\}$

Then,

1. $A \subseteq B$ if and only if

$$
\begin{aligned}
& A_{T}^{-}(x) \leq B_{T}^{-}(x), A_{T}^{+}(x) \leq B_{T}^{+}(x) \\
& A_{I}^{-}(x) \geq B_{I}^{-}(x), A_{I}^{+}(x) \geq B_{I}^{+}(x) \\
& A_{F}^{-}(x) \geq B_{F}^{-}(x), A_{F}^{+}(x) \geq B_{F}^{+}(x) \text { for all } \mathrm{x} \in \mathrm{X} .
\end{aligned}
$$

2. $A=B$ if and only if

$$
\begin{aligned}
& A_{T}^{-}(x)=B_{T}^{-}(x), A_{T}^{+}(x)=B_{T}^{+}(x) \\
& A_{I}^{-}(x)=B_{I}^{-}(x), A_{I}^{+}(x)=B_{I}^{+}(x) \\
& A_{F}^{-}(x)=B_{F}^{-}(x), A_{F}^{+}(x)=B_{F}^{+}(x) \text { for all } \mathrm{x} \in \mathrm{X} .
\end{aligned}
$$

3. $A^{\tilde{C}}=\left\{<x,\left[\mathrm{~A}_{\mathrm{F}}^{-}(\mathrm{x}), \mathrm{A}_{\mathrm{F}}^{+}(\mathrm{x})\right],\left[\mathrm{A}_{\mathrm{I}}^{-}(\mathrm{x}), \mathrm{A}_{\mathrm{I}}^{+}(\mathrm{x})\right],\left[\mathrm{A}_{\mathrm{T}}^{-}(\mathrm{x}), \mathrm{A}_{\mathrm{T}}^{+}(\mathrm{x})\right]>: \mathrm{x} \in \mathrm{X}\right\}$

4. $A \tilde{\cap} B=\left\{<x,\left[\min \left\{\mathrm{A}_{\mathrm{T}}^{-}(\mathrm{x}), \mathrm{B}_{\mathrm{T}}^{-}(\mathrm{x})\right\}, \min \left\{\mathrm{A}_{\mathrm{T}}^{+}(\mathrm{x}), \mathrm{B}_{\mathrm{T}}^{+}(\mathrm{x})\right\}\right]\right.$,

$\left[\max \left\{\mathrm{A}_{\mathrm{I}}^{-}(\mathrm{x}), \mathrm{B}_{\mathrm{I}}^{-}(\mathrm{x})\right\}, \max \left\{\mathrm{A}_{\mathrm{I}}^{+}(\mathrm{x}), \mathrm{B}_{\mathrm{I}}^{+}(\mathrm{x})\right\}\right]$,

$\left.\left[\max \left\{\mathrm{A}_{\mathrm{F}}^{-}(\mathrm{x}), \mathrm{B}_{\mathrm{F}}^{-}(\mathrm{x})\right\}, \max \left\{\mathrm{A}_{\mathrm{F}}^{+}(\mathrm{x}), \mathrm{B}_{\mathrm{F}}^{+}(\mathrm{x})\right\}\right]>: \mathrm{x} \in \mathrm{X}\right\}$

5. $A \tilde{U} B=\left\{<x,\left[\max \left\{\mathrm{A}_{\mathrm{T}}^{-}(\mathrm{x}), \mathrm{B}_{\mathrm{T}}^{-}(\mathrm{x})\right\}, \max \left\{\mathrm{A}_{\mathrm{T}}^{+}(\mathrm{x}), \mathrm{B}_{\mathrm{T}}^{+}(\mathrm{x})\right\}\right]\right.$, $\left[\min \left\{\mathrm{A}_{\mathrm{I}}^{-}(\mathrm{x}), \mathrm{B}_{\mathrm{I}}^{-}(\mathrm{x})\right\}, \min \left\{\mathrm{A}_{\mathrm{I}}^{+}(\mathrm{x}), \mathrm{B}_{\mathrm{I}}^{+}(\mathrm{x})\right\}\right]$, $\left.\left[\min \left\{\mathrm{A}_{\mathrm{F}}^{-}(\mathrm{x}), \mathrm{B}_{\mathrm{F}}^{-}(\mathrm{x})\right\}, \min \left\{\mathrm{A}_{\mathrm{F}}^{+}(\mathrm{x}), \mathrm{B}_{\mathrm{F}}^{+}(\mathrm{x})\right\}\right]>: \mathrm{x} \in \mathrm{X}\right\}$

\subsection{Definition: [1]}

Let $\mathrm{X}$ be an initial universe set. Let $\mathrm{NC}(\mathrm{X})$ denote the set of all neutrosophic cubic sets and $E$ be the set of parameters. Let $A \subset E$ then $\quad(P, A)=\left\{P\left(e_{i}\right)=\quad\left\{<x, A e_{i}(x), \lambda e_{i}(x)>: x \in X\right\} e_{i} \in A\right\}, \quad$ where
More on R-Union and R-Intersection of Neutrosophic Soft Cubic Set 
Cruz, RA Irudayam, FN

$A e_{i}(x)=\left\{<x, A_{e_{i}}^{T}(x), A_{e_{i}}^{I}(x), A_{e_{i}}^{F} \backslash(x)>/ x \in X\right\}$, is an interval neutrosophic set, $\lambda_{e_{i}}(x)=\left\{<x,\left(\lambda_{e_{i}}^{T}(x), \lambda_{e_{i}}^{I}(x), \lambda_{e_{i}}^{F}(x)>/ x \in X\right\}\right.$ is a neutrosophic set. The pair $(P, A)$ is termed to be the neutrosophic soft cubic set over $\mathrm{X}$ where $\mathrm{P}$ is a mapping given by $p: \mathrm{A} \rightarrow \mathrm{NC}(\mathrm{X})$.

\subsection{Definition: [1]}

Let $X$ be an initial universe set. A neutrosophic soft cubic set $(P, M)$ in $\mathrm{X}$ is said to be

- truth-internal (briefly, T-internal) if the following inequality is valid

$$
\left(\forall x \in X, e_{i} \in E\right)\left(A_{e_{i}}^{-T}(x) \leq \lambda_{e_{i}}^{T}(x) \leq A_{e_{i}}^{+T}(x)\right),
$$

- indeterminacy-internal (briefly, I-internal) if the following inequality is valid

$$
\left(\forall x \in X, e_{i} \in E\right)\left(A_{e_{i}}^{-I}(x) \leq \lambda_{e_{i}}^{I}(x) \leq A_{e_{i}}^{+I}(x)\right),
$$

- falsity-internal (briefly, F-internal) if the following inequality is valid

$$
\left(\forall x \in X, e_{i} \in E\right)\left(A_{e_{i}}^{-F}(x) \leq \lambda_{e_{i}}^{F}(x) \leq A_{e_{i}}^{+F}(x)\right) .
$$

If a neutrosophic soft cubic set in $X$ satisfies (2.1), (2.2) and (2.3) we say that $(P, M)$ is an internal neutrosophic soft cubic in $X$.

\subsection{Definition: [1]}

Let $X$ an initial universe set. A neutrosophic soft cubic set $(P, M)$ in $X$ is said to be

- $\quad$ truth-external (briefly, $T$-external) if the following inequality is valid

$$
\left(\forall x \in X, e_{i} \in E\right)\left(\lambda_{e_{i}}^{T}(x) \notin\left(A_{e_{i}}^{-T}(x), A_{e_{i}}^{+T}(x)\right)\right),
$$

- indeterminacy-external (briefly, $I$-external) if the following inequality is valid

$$
\left(\forall x \in X, e_{i} \in E\right)\left(\lambda_{e_{i}}^{I}(x) \notin\left(A_{e_{i}}^{-I}(x), A_{e_{i}}^{+I}(x)\right)\right),
$$

- falsity-external (briefly, $F$-external) if the following inequality is valid

$$
\left(\forall x \in X, e_{i} \in E\right) \quad\left(\lambda_{e_{i}}^{F}(x) \notin\left(A_{e_{i}}^{-F}(x), A_{e_{i}}^{+F}(x)\right)\right) .
$$

If a neutrosophic soft cubic set $(P, M)$ in $X$ satisfies (2.4), (2.5) and (2.6), we say that $(P, M)$ is an external neutrosophic soft cubic in $X$. 


\subsection{Definition [1]}

Let $(P, M)=\left\{P\left(e_{i}\right)=\left\{<x, A_{e_{i}}(x), \lambda_{e_{i}}(x)>: x \in X\right\} e_{i} \in M\right\}$

and $(\mathrm{Q}, \mathrm{N})=\left\{\mathrm{Q}\left(\mathrm{e}_{\mathrm{i}}\right)=\mathrm{B}_{\mathrm{i}}=\left\{<\mathrm{x}, \mathrm{B}_{\mathrm{e}_{\mathrm{i}}}(\mathrm{x}), \mu_{\mathrm{e}_{\mathrm{i}}}(\mathrm{x})>: \mathrm{x} \in \mathrm{X}\right\} \mathrm{e}_{\mathrm{i}} \in \mathrm{N}\right\}$ be two neutrosophic soft cubic sets in $\mathrm{X}$. Let $\mathrm{M}$ and $\mathrm{N}$ be any two subsets of $\mathrm{E}$ (set of parameters), then we have the following

1. $(\mathrm{P}, \mathrm{M})=(\mathrm{Q}, \mathrm{N})$ if and only if the following conditions are satisfied
a) $\mathrm{M}=\mathrm{N}$ and
b) $\mathrm{P}\left(\mathrm{e}_{\mathrm{i}}\right)=\mathrm{Q}\left(\mathrm{e}_{\mathrm{i}}\right)$ for all $e_{i} \in M$ if and only if $A e_{i}(x)=B e_{i}(x)$ and
$\lambda_{e_{i}}(x)=\mu_{e_{i}}(x)$ for all $x \in X$ corresponding to each $e_{i} \in M$.

2. $(\mathrm{P}, \mathrm{M})$ and $(\mathrm{Q}, \mathrm{N})$ are two neutrosophic soft cubic set then we define and denote $\mathrm{P}$ - order as $(\mathrm{P}, \mathrm{M}) \subseteq_{\mathrm{P}}(\mathrm{Q}, \mathrm{N})$ if and only if the following conditions are satisfied

c) $\mathrm{M} \subseteq \mathrm{N}$ and
d) $\quad \mathrm{P}\left(\mathrm{e}_{\mathrm{i}}\right) \leq_{\mathrm{P}} \mathrm{Q}\left(\mathrm{e}_{\mathrm{i}}\right)$ for all $e_{i} \in M$ if and only if $A e_{i}(x) \subseteq B_{e_{i}}(x)$ and $\lambda_{e_{i}}(x) " \mu_{e_{i}}(x)$ for all $x \in X$ corresponding to each $e_{i} \in M$.

3. (P,M) and $(\mathrm{Q}, \mathrm{N})$ are two neutrosophic soft cubic set then we define and denote $\mathrm{P}$ - order as $(\mathrm{P}, \mathrm{M}) \subseteq_{\mathrm{R}}(\mathrm{Q}, \mathrm{N})$ if and only if the following conditions are satisfied

e) $\mathrm{M} \subseteq \mathrm{N}$ and

f) $\mathrm{P}\left(\mathrm{e}_{\mathrm{i}}\right) \leq_{\mathrm{R}} \mathrm{Q}\left(\mathrm{e}_{\mathrm{i}}\right)$ for all $e_{i} \in M$ if and only if $A e_{i}(x) \subseteq B e_{i}(x)$ and $\lambda_{e_{i}}(x) \geq \mu e_{i}(x)$ for all $x \in X$ corresponding to each $e_{i} \in M$.

\subsection{Definition: [1]}

Let $(\mathrm{P}, \mathrm{M})$ and $(\mathrm{Q}, \mathrm{N})$ be two neutrosophic soft cubic sets (NSCS) in X where I and $\mathrm{J}$ are any two subsets of the parametric set $\mathrm{E}$. Then we define R-union of neutrosophic soft cubic set as $(P, M) \cup_{R}(Q, N)=(H, C)$ where $C=M \cup N$

$$
H\left(e_{i}\right)=\left\{\begin{array}{lr}
P\left(e_{i}\right) & \text { if } e_{i} \in M-N \\
Q\left(e_{i}\right) & \text { if } e_{i} \in N-M \\
P\left(e_{i}\right) \vee_{R} Q\left(e_{i}\right) & \text { if } e_{i} \in M \cap N
\end{array}\right\}
$$

where $P\left(e_{i}\right) \vee_{R} Q\left(e_{i}\right)$ is defined as

$$
\begin{aligned}
& P\left(e_{i}\right) \vee_{R} Q\left(e_{i}\right) \\
&\left\{<\mathrm{x}, \max \left\{\mathrm{A}_{\mathrm{e}_{\mathrm{i}}}(\mathrm{x}), \mathrm{B}_{\mathrm{e}_{\mathrm{i}}}(\mathrm{x})\right\},\left(\lambda \wedge \mu_{\mathrm{e}_{\mathrm{i}}}\right)(\mathrm{x})>: \mathrm{x} \in \mathrm{X}\right\} \mathrm{e}_{\mathrm{i}} \in \mathrm{M} \cap \mathrm{N} \\
& \text { where } \mathrm{A}_{\mathrm{e}_{\mathrm{i}}}(\mathrm{x}), \mathrm{B}_{\mathrm{e}_{\mathrm{i}}}(\mathrm{x}) \text { represent interval neutrosophic sets. Hence }
\end{aligned}
$$

More on R-Union and R-Intersection of Neutrosophic Soft Cubic Set 
Cruz, RA

Irudayam, FN

$$
\begin{aligned}
& P^{T}\left(e_{i}\right) \vee_{R} Q^{T}\left(e_{i}\right)=\left\{<\mathrm{x}, \max \left\{\mathrm{A}_{\mathrm{e}_{\mathrm{i}}}^{\mathrm{T}}(\mathrm{x}), \mathrm{B}_{\mathrm{e}_{\mathrm{i}}^{\mathrm{T}}}^{\mathrm{T}}(\mathrm{x})\right\},\left(\lambda_{\mathrm{e}_{\mathrm{i}}}^{\mathrm{T}} \wedge \mu_{\mathrm{e}_{\mathrm{i}}}^{\mathrm{T}}\right)(\mathrm{x})>: \mathrm{x} \in \mathrm{X}\right\} \\
& \mathrm{e}_{\mathrm{i}} \in \mathrm{M} \cap \mathrm{N}, \\
& P^{I}\left(e_{i}\right) \vee_{R} Q^{\mathrm{I}}\left(e_{i}\right)=\left\{<\mathrm{x}, \max \left\{\mathrm{A}_{\mathrm{e}_{\mathrm{i}}}^{\mathrm{I}}(\mathrm{x}), \mathrm{B}_{\mathrm{e}_{\mathrm{i}}}^{\mathrm{I}}(\mathrm{x})\right\},\left(\lambda_{\mathrm{e}_{\mathrm{i}}}^{I} \wedge \mu_{\mathrm{e}_{\mathrm{i}}}^{I}\right)(\mathrm{x})>: \mathrm{x} \in \mathrm{X}\right\} \\
& \mathrm{e}_{\mathrm{i}} \in \mathrm{M} \cap \mathrm{N}, \\
& P^{F}\left(e_{i}\right) \vee_{R} Q^{F}\left(e_{i}\right)=\left\{<\mathrm{x}, \max \left\{\mathrm{A}_{\mathrm{e}_{\mathrm{i}}}^{\mathrm{F}}(\mathrm{x}), \mathrm{B}_{\mathrm{e}_{\mathrm{i}}}^{\mathrm{F}}(\mathrm{x})\right\},\left(\lambda_{\mathrm{e}_{\mathrm{i}}}^{\mathrm{F}} \wedge \mu_{\mathrm{e}_{\mathrm{i}}^{\mathrm{F}}}^{\mathrm{F}}\right)(\mathrm{x})>: \mathrm{x} \in \mathrm{X}\right\} \\
& \mathrm{e}_{\mathrm{i}} \in \mathrm{M} \cap \mathrm{N} .
\end{aligned}
$$

\subsection{Definition: [1]}

Let $(\mathrm{P}, \mathrm{M})$ and $(\mathrm{Q}, \mathrm{N})$ be two neutrosophic soft cubic sets (NSCS) in X where $\mathrm{M}$ and $\mathrm{N}$ are any subsets of parameter's set E.

Then we define R-intersection of neutrosophic soft cubic set as $(P, M) \cap_{R}(Q, N)=(H, C)$ where $C=M \cap N$,

$H\left(e_{i}\right)=P\left(e_{i}\right) \wedge_{R} Q\left(e_{i}\right)$

$H\left(e_{i}\right)=P\left(e_{i}\right) \wedge_{R} Q\left(e_{i}\right)$ and $e_{i} \in I \cap J$.Here $F\left(e_{i}\right) \wedge_{R} G\left(e_{i}\right)$ is defined as

$$
\begin{aligned}
& P\left(e_{i}\right) \wedge_{R} Q\left(e_{i}\right)=H\left(e_{i}\right)=\left\{<\mathrm{x}, \min \left\{\mathrm{A}_{\mathrm{e}_{\mathrm{i}}}(\mathrm{x}), \mathrm{B}_{\mathrm{e}_{\mathrm{i}}}(\mathrm{x})\right\},\left(\lambda_{\mathrm{e}_{\mathrm{i}}} \vee \mu_{\mathrm{e}_{\mathrm{i}}}\right)(\mathrm{x})>: \mathrm{x} \in \mathrm{X}\right\} \\
& \mathrm{e}_{\mathrm{i}} \in \mathrm{M} \cap \mathrm{N} .
\end{aligned}
$$

where $\mathrm{A}_{\mathrm{e}_{\mathrm{i}}}(\mathrm{x}), \mathrm{B}_{\mathrm{e}_{\mathrm{i}}}(\mathrm{x})$ represent interval neutrosophic sets. Hence

$$
\begin{aligned}
& P^{T}\left(e_{i}\right) \wedge_{R} Q^{T}\left(e_{i}\right)=\left\{<\mathrm{x}, \min \left\{\mathrm{A}_{\mathrm{e}_{\mathrm{i}}}^{\mathrm{T}}(\mathrm{x}), \mathrm{B}_{\mathrm{e}_{\mathrm{i}}^{\mathrm{T}}}^{\mathrm{T}}(\mathrm{x})\right\},\left(\lambda_{\mathrm{e}_{\mathrm{i}}}^{T} \vee \mu_{\mathrm{e}_{\mathrm{i}}}^{\mathrm{T}}\right)(\mathrm{x})>: \mathrm{x} \in \mathrm{X}\right\} \\
& \mathrm{e}_{\mathrm{i}} \in \mathrm{M} \cap \mathrm{N}, \\
& P^{I}\left(e_{i}\right) \wedge_{R} Q^{I}\left(e_{i}\right)=\left\{<\mathrm{x}, \min \left\{\mathrm{A}_{\mathrm{e}_{\mathrm{i}}}^{\mathrm{I}}(\mathrm{x}), \mathrm{B}_{\mathrm{e}_{\mathrm{i}}}^{\mathrm{I}}(\mathrm{x})\right\},\left(\lambda_{\mathrm{e}_{\mathrm{i}}}^{\mathrm{I}} \vee \mu_{\mathrm{e}_{\mathrm{i}}}^{\mathrm{I}}\right)(\mathrm{x})>: \mathrm{x} \in \mathrm{X}\right\} \\
& \mathrm{e}_{\mathrm{i}} \in \mathrm{M} \cap \mathrm{N}, \\
& P^{F}\left(e_{i}\right) \wedge_{R} Q^{F}\left(e_{i}\right)=\left\{<\mathrm{x}, \min \left\{\mathrm{A}_{\mathrm{e}_{\mathrm{i}}}^{\mathrm{F}}(\mathrm{x}), \mathrm{B}_{\mathrm{e}_{\mathrm{i}}}^{\mathrm{F}}(\mathrm{x})\right\},\left(\lambda_{\mathrm{e}_{\mathrm{i}}}^{\mathrm{F}} \vee \mu_{\mathrm{e}_{\mathrm{i}}}^{F}\right)(\mathrm{x})>: \mathrm{x} \in \mathrm{X}\right\} \\
& \mathrm{e}_{\mathrm{i}} \in \mathrm{M} \cap \mathrm{N}
\end{aligned}
$$

\subsection{Definition: [2]}

The complement of a neutrosophic soft cubic set $(\mathrm{F}, \mathrm{I})=\left\{\mathrm{F}\left(\mathrm{e}_{\mathrm{i}}\right)=\left\{<\mathrm{x}, \mathrm{A}_{\mathrm{e}_{\mathrm{i}}}(\mathrm{x}), \lambda_{\mathrm{e}_{\mathrm{i}}}(\mathrm{x})>: \mathrm{x} \in \mathrm{X}\right\} \mathrm{e}_{\mathrm{i}} \in \mathrm{I}\right\}$ is denoted by $(\mathrm{F}, \mathrm{I})^{\mathrm{C}}$
and defined as

$\left.(\mathrm{F}, \mathrm{I})^{\mathrm{C}}=(\mathrm{F}, \mathrm{I})^{\mathrm{c}}=\left(\mathrm{F}^{\mathrm{c}}, \neg \mathrm{I}\right)\right\}$, where $F^{c}: \neg I \rightarrow N C(X)$ and

$(\mathrm{F}, \mathrm{I}){ }^{\mathrm{c}}=\left\{\left(\mathrm{F}\left(\mathrm{e}_{\mathrm{i}}\right)\right)^{\mathrm{c}}=\left\{<\mathrm{x}, \mathrm{A}_{e_{i}}^{c}(\mathrm{x}), \lambda_{e_{i}}^{c}(\mathrm{x})>: \mathrm{x} \in \mathrm{X}\right\} \mathrm{e}_{\mathrm{i}} \in \mathrm{I}\right\}$. 
$(\mathrm{F}, \mathrm{I})^{\mathrm{c}}=\left\{<\mathrm{x},\left(\left[1-A_{e_{i}}^{+T}, 1-A_{e_{i}}^{-T}\right],\left[1-A_{e_{i}}^{+I}, 1-A_{e_{i}}^{-I}\right],\left[1-A_{e_{i}}^{+F}, 1-A_{e_{i}}^{-F}\right]\right)\right.$, $\left.\left(1-\lambda_{e_{i}}^{T}, 1-\lambda_{e_{i}}^{I}, 1-\lambda_{e_{i}}^{F}\right)>\mathrm{x} \in \mathrm{X}\right\} \mathrm{e}_{\mathrm{i}} \in \mathrm{I}$.
More on R-Union and R-Intersection of Neutrosophic Soft Cubic Set

\section{MORE ON R-UNION AND R-INTERSECTION OF NEUTROSOPHIC SOFT CUBIC SET}

\section{Definition: 3.1}

Let $(\mathrm{P}, \mathrm{M})=\left\{\mathrm{F}\left(\mathrm{e}_{\mathrm{i}}\right)=\left\{<\mathrm{x}, \mathrm{A}_{\mathrm{e}_{\mathrm{i}}}(\mathrm{x}), \lambda_{\mathrm{e}_{\mathrm{i}}}(\mathrm{x})>: \mathrm{x} \in \mathrm{X}\right\} \mathrm{e}_{\mathrm{i}} \in \mathrm{M}\right\}$ and $(\mathrm{Q}, \mathrm{N})=\left\{\mathrm{G}\left(\mathrm{e}_{\mathrm{i}}\right)=\left\{<\mathrm{x}, \mathrm{B}_{\mathrm{e}_{\mathrm{i}}}(\mathrm{x}), \mu_{\mathrm{e}_{\mathrm{i}}}(\mathrm{x})>: \mathrm{x} \in \mathrm{X}\right\} \mathrm{e}_{\mathrm{i}} \in \mathrm{N}\right\}$ be NSCS in $\mathrm{X}$. Then

1. R-OR of NSCS is denoted by $(P, M) \vee_{R}(Q, N)$ and defined as $(P, M) \vee_{R}(Q, N)=(H, M \times N)$ where $H\left(\alpha_{i}, \beta_{i}\right)=P\left(\alpha_{i}\right) \cup_{R} Q\left(\beta_{i}\right)$ for all $\left(\alpha_{i}, \beta_{i}\right) \in M \times N$.

2. R-AND of NSCS is denoted by $(P, M) \wedge_{R}(Q, N)$ and defined as $(P, M) \wedge_{R}(Q, N)=(H, M \times N)$ where $H\left(\alpha_{i}, \beta_{i}\right)=F\left(\alpha_{i}\right) \cap_{R} G\left(\beta_{i}\right)$ for all $\left(\alpha_{i}, \beta_{i}\right) \in M \times N$.

\section{Example: 3.2}

Let $X=\left\{x_{1}, x_{2}, x_{3}\right\}$ be initial universe and $E=\left\{e_{1}, e_{2}\right\}$ parameter's set. Let $(\mathrm{P}, \mathrm{M})$ be a neutrosophic soft cubic set over $\mathrm{X}$ and defined as $(\mathrm{P}, \mathrm{M})=\left\{\mathrm{P}\left(\mathrm{e}_{\mathrm{i}}\right)=\left\{<\mathrm{x}, \mathrm{A}_{\mathrm{e}_{\mathrm{i}}}(\mathrm{x}), \lambda_{\mathrm{e}_{\mathrm{i}}}(\mathrm{x})>: \mathrm{x} \in \mathrm{X}\right\} \mathrm{e}_{\mathrm{i}} \in \mathrm{M}\right\}$ and

\begin{tabular}{|c|c|c|c|c|}
\hline \multirow{2}{*}{$\mathrm{X}$} & \multicolumn{2}{|c|}{$\mathrm{P}\left(\mathrm{e}_{1}\right)$} & \multicolumn{2}{c|}{$\mathrm{P}\left(\mathrm{e}_{2}\right)$} \\
\cline { 2 - 5 } & $<\mathrm{Ae}_{1}(\mathrm{x})$, & $\lambda \mathrm{e}_{1}(\mathrm{x})>$ & $<\mathrm{Ae}_{2}(\mathrm{x})$, & $\lambda \mathrm{e}_{2}(\mathrm{x})>$ \\
\hline $\mathrm{x}_{1}$ & {$[0.5,0.6][0.6,0.7][0.5,0.6]$} & {$[0.7,0.4,0.6]$} & {$[0.3,0.6][0.2,0.7][0.2,0.4]$} & {$[0.5,0.2,0.2]$} \\
\hline $\mathrm{x}_{2}$ & {$[0.4,0.5][0.7,0.8][0.2,0.3]$} & {$[0.6,0.4,0.2]$} & {$[0.3,0.5][0.6,0.8][0.2,0.6]$} & {$[0.6,0.5,0.4]$} \\
\hline $\mathrm{x}_{3}$ & {$[0.2,0.3][0.2,0.3][0.3,0.5]$} & {$[0.5,0.3,0.5]$} & {$[0.4,0.7][0.2,0.5][0.3,0.6]$} & {$[0.7,0.3,0.4]$} \\
\hline
\end{tabular}

$(\mathrm{Q}, \mathrm{N})=\left\{\mathrm{G}\left(\mathrm{e}_{\mathrm{i}}\right)=\left\{<\mathrm{x}, \mathrm{B}_{\mathrm{e}_{\mathrm{i}}}(\mathrm{x}), \mu_{\mathrm{e}_{\mathrm{i}}}(\mathrm{x})>: \mathrm{x} \in \mathrm{X}\right\} \mathrm{e}_{\mathrm{i}} \in \mathrm{N}\right\}$

\begin{tabular}{|c|c|c|c|c|}
\hline \multirow{2}{*}{$\mathrm{X}$} & \multicolumn{2}{|c|}{$\mathrm{Q}\left(\mathrm{e}_{1}\right)$} & \multicolumn{2}{c|}{$\mathrm{Q}\left(\mathrm{e}_{2}\right)$} \\
\cline { 2 - 5 } & $\left\langle\mathrm{Be}_{1}(\mathrm{x})\right.$, & $\left.\mu \mathrm{e}_{1}(\mathrm{x})\right\rangle$ & $<\mathrm{Ae}_{2}(\mathrm{x})$, & $\left.\mu \mathrm{e}_{2}(\mathrm{x})\right\rangle$ \\
\hline $\mathrm{x}_{1}$ & {$[0.7,0.9][0.3,0.5][0.3,0.4]$} & {$[0.4,0.5,0.6]$} & {$[0.4,0.7][0.1,0.3][0.1,0.2]$} & {$[0.3,0.4,0.4]$} \\
\hline $\mathrm{x}_{2}$ & {$[0.5,0.6][0.3,0.7][0.1,0.2]$} & {$[0.5,0.6,0.6]$} & {$[0.4,0.6][0.4,0.7][0.2,0.5]$} & {$[0.4,0.7,0.5]$} \\
\hline $\mathrm{x}_{3}$ & {$[0.3,0.4][0.1,0.2][0.2,0.4]$} & {$[0.3,0.4,0.6]$} & {$[0.5,0.8][0.1,0.4][0.1,0.4]$} & {$[0.5,0.6,0.6]$} \\
\hline
\end{tabular}

$\mathrm{R}$-OR is denoted by $(H, M \times N)=(P, M) \vee_{R}(Q, N)$ where $M \times N=\left\{\left(\mathrm{e}_{1}, \mathrm{e}_{1}\right),\left(\mathrm{e}_{1}, \mathrm{e}_{2}\right),\left(\mathrm{e}_{2}, \mathrm{e}_{1}\right),\left(\mathrm{e}_{2}, \mathrm{e}_{2}\right)\right\}$ is defined 
Cruz, RA

Irudayam, FN

\begin{tabular}{|c|c|c|c|c|c|c|c|c|}
\hline $\mathrm{X}$ & \multicolumn{2}{|c|}{$\mathrm{H}\left(\mathrm{e}_{1}, \mathrm{e}_{1}\right)$} & \multicolumn{2}{c|}{$\mathrm{H}\left(\mathrm{e}_{1}, \mathrm{e}_{2}\right)$} & \multicolumn{2}{c|}{$\mathrm{H}\left(\mathrm{e}_{2}, \mathrm{e}_{1}\right)$} & \multicolumn{2}{c|}{$\mathrm{H}\left(\mathrm{e}_{2}, \mathrm{e}_{2}\right)$} \\
\cline { 2 - 9 } & \multicolumn{2}{|c|}{$P\left(e_{1}\right) \cap Q\left(e_{1}\right)$} & \multicolumn{2}{c|}{$P\left(e_{1}\right) \cap Q\left(e_{2}\right)$} & \multicolumn{2}{c|}{$P\left(e_{2}\right) \cap Q\left(e_{1}\right)$} & \multicolumn{2}{c|}{$P\left(e_{2}\right) \cap Q\left(e_{2}\right)$} \\
\hline $\mathrm{x}_{1}$ & {$[0.7,0.9]$} & {$[0.4,0.4,0.6]$} & {$[0.5,0.6]$} & {$[0.3,0.4,0.4]$} & {$[0.7,0.9]$} & {$[0.4,0.2,0.2]$} & {$[0.4,00.7]$} & {$[0.3,0.2,0.2]$} \\
& {$[0.6,0.7]$} & & {$[0.6,0.7]$} & & {$[0.3,0.5]$} & & {$[0.2,0.7]$} & \\
& {$[0.5,0.6]$} & & {$[0.5,0.6]$} & & {$[0.3,0.4]$} & & {$[0.2,0.4]$} & \\
\hline $\mathrm{x}_{2}$ & {$[0.5,0.6]$} & {$[0.5,0.4,0.2]$} & {$[0.4,0.6]$} & {$[0.4,0.4,0.2]$} & {$[0.5,0.6]$} & {$[0.5,0.5,0.4]$} & {$[0.4,0.6]$} & {$[0.4,0.5,0.4]$} \\
& {$[0.7,0.8]$} & & {$[0.7,0.8]$} & & {$[0.6,0.8]$} & & {$[0.6,0.8]$} & \\
& {$[0.2,0.3]$} & & {$[0.2,0.5]$} & & {$[0.2,0.6]$} & & {$[0.2,0.6]$} & \\
\hline $\mathrm{x}_{3}$ & {$[0.3,0.4]$} & {$[035,0.3,0.5]$} & {$[0.5,0.8]$} & {$[0.5,0.3,0.5]$} & {$[0.4,0.7]$} & {$[0.3,0.3,0.4]$} & {$[0.5,0.8]$} & {$[0.5,0.3,0.4]$} \\
& {$[0.2,0.3]$} & & {$[0.2,0.3]$} & & {$[0.2,05]$} & & {$[0.2,0.5]$} & \\
& {$[0.3,0.5]$} & & {$[0.3,0.5]$} & & {$[0.3,06]$} & & {$[0.3,0.6]$} & \\
\hline
\end{tabular}

R-AND is denoted by $(H, M \times N)=(P, M) \wedge_{R}(Q, N)$ where $M \times N=\left\{\left(\mathrm{e}_{1}, \mathrm{e}_{1}\right),\left(\mathrm{e}_{1}, \mathrm{e}_{2}\right),\left(\mathrm{e}_{2}, \mathrm{e}_{1}\right),\left(\mathrm{e}_{2}, \mathrm{e}_{2}\right)\right\}$ is defined

\begin{tabular}{|c|c|c|c|c|c|c|c|c|}
\hline $\mathrm{x}$ & \multicolumn{2}{|c|}{$\mathrm{H}\left(\mathrm{e}_{1}, \mathrm{e}_{1}\right)$} & \multicolumn{2}{c|}{$\mathrm{H}\left(\mathrm{e}_{1}, \mathrm{e}_{2}\right)$} & \multicolumn{2}{c|}{$\mathrm{H}\left(\mathrm{e}_{2}, \mathrm{e}_{1}\right)$} & \multicolumn{2}{c|}{$\mathrm{H}\left(\mathrm{e}_{2}, \mathrm{e}_{2}\right)$} \\
\cline { 2 - 8 } & \multicolumn{2}{|c|}{$P\left(e_{1}\right) \cap Q\left(e_{1}\right)$} & \multicolumn{2}{|c|}{$P\left(e_{1}\right) \cap Q\left(e_{2}\right)$} & \multicolumn{2}{c|}{$P\left(e_{2}\right) \cap Q\left(e_{1}\right)$} & \multicolumn{2}{|c|}{$P\left(e_{2}\right) \cap Q\left(e_{2}\right)$} \\
\hline $\mathrm{x}_{1}$ & {$[0,5,0.6]$} & {$[0.7,0.5,0.6]$} & {$[0.4,0.7]$} & {$[0.5,0.5,0.6]$} & {$[0.3,0.6]$} & {$[0.7,0.4,0.5]$} & {$[0.3,0.6]$} & {$[0.5,0.4,0.4]$} \\
& {$[0.3,0.5]$} & & {$[0.1,0.3]$} & & {$[0.2,0.7]$} & & {$[0.1,0.3]$} & \\
& {$[0.3,0.4]$} & & {$[0.1,0.2]$} & & {$[0.2,0.4]$} & & {$[0.1,0.2]$} & \\
\hline $\mathrm{x}_{2}$ & {$[0.4,0.5]$} & {$[0 . .6,0.6,0.6]$} & {$[0.4,0.5]$} & {$[[0.6,0.6,0.6]$} & {$[0.3,0.5]$} & {$[0 . .6,0.7,0.5]$} & {$[0.3,0.5]$} & {$[0.6,0.7,0.5]$} \\
& {$[0.3,0.7]$} & & {$[0.4,0.7]$} & & {$[0.3,0.7]$} & & {$[0.4,0.7]$} & \\
& {$[0.1,0.2]$} & & {$[0.2,0.3]$} & & {$[0.1,0.2]$} & & {$[0.2,0.5]$} & \\
\hline $\mathrm{x}_{3}$ & {$[0.2,0.3]$} & {$[0.5,0.4,0.6]$} & {$[0.2,0.3]$} & {$[0.7,0.4,0.6]$} & {$[0.3,0.4]$} & {$[0.5,0.6,0.6]$} & {$[0.4,0.7]$} & {$[0.7,0.6,0.6]$} \\
& {$[0.1,0.2]$} & & {$[0.1,0.4]$} & & {$[0.1,0.2]$} & & {$[0.1,0.4]$} & \\
& {$[0.2,0.4]$} & & {$[0.1,0.4]$} & & {$[0.2,0.4]$} & & {$[0.1,0.4]$} & \\
\hline
\end{tabular}

Proposition: 3.3 Let $\mathrm{X}$ be initial universe and I,J,L and $\mathrm{S}$ subsets of $\mathrm{E}$. Then for any neutrosophic soft cubic sets $\mathcal{A}=(F, I), \mathcal{B}=(G, J), C=(E, L), \mathcal{D}=(T, S)$ the following properties hold

(1) if $\mathcal{A} \subseteq_{R} \mathcal{B}$ and $\mathcal{B} \subseteq_{R} C$ then $\mathcal{A} \subseteq_{R} C$.

(2) if $\mathcal{A} \subseteq_{R} \mathcal{B}$ then $\mathcal{B}^{c} \subseteq_{R} \mathcal{A}^{c}$.

(3) if $\mathcal{A} \subseteq_{R} \mathcal{B}$ and $\mathcal{A} \subseteq_{R} C$ then $\mathcal{A} \subseteq_{R} \mathcal{B} \cap_{R} C$.

(4) if $\mathcal{A} \subseteq_{R} \mathcal{B}$ and $C \subseteq_{R} \mathcal{B}$ then $\mathcal{A} \cup_{R} C \subseteq_{R} \mathcal{B}$.

(5) if $\mathcal{A} \subseteq_{R} \mathcal{B}$ and $C \subseteq_{R} \mathcal{D}$ then $\mathcal{A} \cup_{R} C \subseteq_{R} \mathcal{B} \cup_{R} \mathcal{D}$ and $\mathcal{A} \cap_{R} C \subseteq_{R}$ $\mathcal{B} \cup_{R} \mathcal{D}$.

Proof: Straight forward. 


\section{Theorem: 3.4}

Let $(\mathrm{P}, \mathrm{M})$ and $(\mathrm{Q}, \mathrm{N})$ be INSCS over $\mathrm{X}$ such that $\max \left\{A_{e_{i}}^{-T}(x), B_{e_{i}}^{-T}(x)\right\} \leq\left(\lambda_{e_{\mathrm{i}}}^{T} \wedge \mu_{\mathrm{e}_{\mathrm{i}}}^{\mathrm{T}}\right)(x), \max \left\{A_{e_{i}}^{-I}(x), B_{e_{i}}^{-I}(x)\right\} \leq\left(\lambda_{\mathrm{e}_{\mathrm{i}}}^{I} \wedge \mu_{\mathrm{e}_{\mathrm{i}}}^{\mathrm{I}}\right)$, $\max \left\{A_{e_{i}}^{-F}(x), B_{e_{i}}^{-F}(x)\right\} \leq\left(\lambda_{\mathrm{e}_{\mathrm{i}}}^{F} \wedge \mu_{\mathrm{e}_{\mathrm{i}}}^{\mathrm{F}}\right)(x)$ for all $\mathrm{e}_{\mathrm{i}} \in \mathrm{M} \cap \mathrm{N}$ and for all $\mathrm{x} \in \mathrm{X}$, then $(\mathrm{P}, \mathrm{M}) \cup_{R}(Q, N)$ is also an INSCS.

Proof:

Since $(\mathrm{P}, \mathrm{M})$ and $(\mathrm{Q}, \mathrm{N})$ is an INSCS.

So far $(\mathrm{P}, \mathrm{M})$ we have $A_{e_{i}}^{-T}(x) \leq \lambda_{e_{i}}^{T}(x) \leq A_{e_{i}}^{+T}(x), A_{e_{i}}^{-I}(x) \leq \lambda_{e_{i}}^{I}(x) \leq A_{e_{i}}^{+I}(x)$, $A_{e_{i}}^{-F}(x) \leq \lambda_{e_{i}}^{F}(x) \leq A_{e_{i}}^{+F}(x)$ for all $\mathrm{e}_{\mathrm{i}} \in \mathrm{M}$ and for all $\mathrm{x} \in \mathrm{X}$.

And for $(\mathrm{Q}, \mathrm{N})$ we have $B_{e_{i}}^{-T}(x) \leq \mu_{e_{i}}^{T}(x) \leq B_{e_{i}}^{+T}(x) \quad B_{e_{i}}^{-I}(x) \leq \mu_{e_{i}}^{I}(x) \leq B_{e_{i}}^{+I}(x)$, $B_{e_{i}}^{-F}(x) \leq \mu_{e_{i}}^{F}(x) \leq B_{e_{i}}^{+F}(x)$ for all $\mathrm{e}_{\mathrm{i}} \in \mathrm{N}$ and for all $\mathrm{x} \in \mathrm{X}$.

$$
\begin{aligned}
& \left(\underset{e_{\mathrm{i}}}{\lambda^{T}} \wedge \mu_{e_{\mathrm{i}}}^{\mathrm{T}}\right)(x) \leq \max \left\{A_{e_{i}}^{+T}(x), B_{e_{i}}^{+T}(x)\right\},\left(\lambda_{\mathrm{e}_{\mathrm{i}}}^{I} \wedge \mu_{\mathrm{e}_{\mathrm{i}}}^{\mathrm{I}}\right)(x) \leq \max \left\{A_{e_{i}}^{+I}(x), B_{e_{i}}^{+I}(x)\right\}, \\
& \left(\underset{\mathrm{e}_{\mathrm{i}}}{\lambda_{F}^{F}} \wedge \mu_{\mathrm{e}_{\mathrm{i}}}^{\mathrm{F}}\right)(x) \leq \max \left\{A_{e_{i}}^{+F}(x), B_{e_{i}}^{+F}(x)\right\}
\end{aligned}
$$

for all $\mathrm{e}_{\mathrm{i}} \in \mathrm{M}$ and for all $\mathrm{x} \in \mathrm{X}$. Also given that $\max \left\{A_{e_{i}}^{-T}(x), B_{e_{i}}^{-T}(x)\right\} \leq$ $\left(\lambda_{\mathrm{e}_{\mathrm{i}}}^{T} \wedge \mu_{\mathrm{e}_{\mathrm{i}}}^{\mathrm{T}}\right)(x), \max \left\{A_{e_{i}}^{-I}(x), B_{e_{i}}^{-I}(x)\right\} \leq\left(\lambda_{\mathrm{e}_{\mathrm{i}}}^{I} \wedge \mu_{\mathrm{e}_{\mathrm{i}}}^{\mathrm{I}}\right), \max \left\{A_{e_{i}}^{-F}(x), B_{e_{i}}^{-F}(x)\right\} \leq$ $\left(\lambda_{\mathrm{e}_{\mathrm{i}}}^{F} \wedge \mu_{\mathrm{e}_{\mathrm{i}}}^{\mathrm{F}}\right)(x)$ for all $\mathrm{e}_{\mathrm{i}} \in \mathrm{M} \cap \mathrm{N}$ and for all $\mathrm{x} \in \mathrm{X}$. Now $(\mathrm{P}, \mathrm{M}) \cup_{\mathrm{R}}$ $(Q, N)=(H, C)$ where $\mathrm{M} \cup N=C$ and

$$
H\left(e_{i}\right)=\left\{\begin{array}{lr}
P\left(e_{i}\right) & \text { if } e \in M-N \\
Q\left(e_{i}\right) & \text { if } e \in N-M \\
P\left(e_{i}\right) \vee_{R} Q\left(e_{i}\right) & \text { if } e \in M \cap N
\end{array}\right\}
$$

If $e \in M \cap N$, then $P\left(e_{i}\right) \vee_{R} Q\left(e_{i}\right)$ is defined as

$P\left(e_{i}\right) \vee_{R} Q\left(e_{i}\right)=H\left(e_{i}\right)=\left\{<x, \max \left\{A_{e_{i}}(x), B_{e_{i}}(x)\right\},\left(\lambda_{e_{i}} \wedge \mu_{e_{i}}\right)(x), x \in X\right.$,

$\left.e_{i} \in M \cap N\right\}$

where

$$
\begin{aligned}
& P^{T}\left(e_{i}\right) \vee_{R} Q^{T}\left(e_{i}\right)=\left\{<x, \max \left\{A_{e_{i}}^{T}(x), B_{e_{i}}^{T}(x)\right\},\left(\lambda_{e_{\mathrm{i}}}^{T} \wedge \mu_{\mathrm{e}_{\mathrm{i}}}^{\mathrm{T}}\right)(x), x \in X,\right. \\
& \left.e_{i} \in M \cap N\right\}
\end{aligned}
$$

More on R-Union and R-Intersection of Neutrosophic Soft Cubic Set 
Cruz, RA

Irudayam, FN

$$
\begin{aligned}
& P^{I}\left(e_{i}\right) \vee_{R} Q^{I}\left(e_{i}\right)=\left\{<x, \max \left\{A_{e_{i}}^{I}(x), B_{e_{i}}^{I}(x)\right\},\left(\lambda_{\mathrm{e}_{\mathrm{i}}}^{I} \wedge \mu_{\mathrm{e}_{\mathrm{i}}}^{\mathrm{I}}\right)(x), x \in X,\right. \\
& \left.e_{i} \in M \cap N\right\}, \\
& P^{F}\left(e_{i}\right) \vee_{R} Q^{F}\left(e_{i}\right)=\left\{<x, \max \left\{A_{e_{i}}^{F}(x), B_{e_{i}}^{F}(x)\right\},\left(\lambda_{\mathrm{e}_{\mathrm{i}}}^{F} \wedge \mu_{\mathrm{e}_{\mathrm{i}}}^{\mathrm{F}}\right)(x), x \in X,\right.
\end{aligned}
$$

$\left.e_{i} \in M \cap N\right\}$

Since $(\mathrm{P}, \mathrm{M})$ and $(\mathrm{Q}, \mathrm{N})$ are INSCS so from above given condition and definition of an INSCS we can write, $\max \left\{A_{e_{i}}^{-T}(x), B_{e_{i}}^{-T}(x)\right\} \leq\left(\lambda_{\mathrm{e}_{\mathrm{i}}}^{T} \wedge \mu_{\mathrm{e}_{\mathrm{i}}}^{\mathrm{T}}\right)(x) \leq \max \left\{A_{e_{i}}^{+T}(x)\right.$, ,$\left.B_{e_{i}}^{+T}(x)\right\}, \max \left\{A_{e_{i}}^{-I}(x), B_{e_{i}}^{-I}(x)\right\} \leq\left(\lambda_{\mathrm{e}_{\mathrm{i}}}^{I} \wedge \mu_{\mathrm{e}_{\mathrm{i}}}^{\mathrm{I}}\right)(x) \leq \max \left\{A_{e_{i}}^{+I}(x), B_{e_{i}}^{+I}(x)\right\}$ $\max \left\{A_{e_{i}}^{-F}(x), B_{e_{i}}^{-F}(x)\right\} \leq\left(\lambda_{e_{i}}^{F} \wedge \mu_{e_{i}}^{\mathrm{F}}\right)(x) \leq \max \left\{A_{e_{i}}^{+F}(x), B_{e_{i}}^{+F}(x)\right\}$ for all $\mathrm{e}_{\mathrm{i}} \in \mathrm{M} \cap N$ and for all $\mathrm{x} \in \mathrm{X}^{\mathrm{i}}$. If $\mathrm{e}_{\mathrm{i}} \in \mathrm{M}-N$ or $\mathrm{e}_{\mathrm{i}} \in N-M$ then the result is trivial. Thus $(\mathrm{P}, \mathrm{M}) \cup_{R}(Q, N)=(H, C)$ is an INSCS if that $\max \left\{A_{e_{i}}^{-T}(x), B_{e_{i}}^{-T}\right.$ $(x)\} \leq\left(\lambda_{\mathrm{e}_{\mathrm{i}}}^{T} \wedge \mu_{\mathrm{e}_{\mathrm{i}}}^{\mathrm{T}}\right)(x), \max \left\{A_{e_{i}}^{-I}(x), B_{e_{i}}^{-I}(x)\right\} \leq\left(\lambda_{\mathrm{e}_{\mathrm{i}}}^{I} \wedge \mu_{\mathrm{e}_{\mathrm{i}}}^{\mathrm{I}}\right)(\mathrm{x}), \max \left\{A_{e_{i}}^{-F}(x)\right.$,
$\left.B_{e_{i}}^{-F}(x)\right\} \leq\left(\lambda_{\mathrm{e}_{\mathrm{i}}}^{F} \wedge \mu_{\mathrm{e}_{\mathrm{i}}}^{\mathrm{F}}\right)(x)$.

\section{Theorem 3.5}

Let $(\mathrm{P}, \mathrm{M})=\left\{\mathrm{P}\left(\mathrm{e}_{\mathrm{i}}\right)=\left\{<\mathrm{x}, \mathrm{A}_{\mathrm{e}_{\mathrm{i}}}(\mathrm{x}), \lambda_{\mathrm{e}_{\mathrm{i}}}(\mathrm{x})>: \mathrm{x} \in \mathrm{X}\right\} \mathrm{e}_{\mathrm{i}} \in \mathrm{M}\right\}$ and

$(\mathrm{Q}, \mathrm{N})=\left\{\mathrm{Q}\left(\mathrm{e}_{\mathrm{i}}\right)=\left\{<\mathrm{x}, \mathrm{B}_{\mathrm{e}_{\mathrm{i}}}(\mathrm{x}), \mu_{\mathrm{e}_{\mathrm{i}}}(\mathrm{x})>: \mathrm{x} \in \mathrm{X}\right\} \mathrm{e}_{\mathrm{i}} \in \mathrm{N}\right\}$ be INSCS in $\mathrm{X}$ satisfying the following inequality $\min \left\{A_{e_{i}}^{+T}(x), B_{e_{i}}^{+T}(x)\right\} \geq\left(\lambda_{\mathrm{e}_{\mathrm{i}}}^{T} \vee \mu_{\mathrm{e}_{\mathrm{i}}}^{\mathrm{T}}\right)(x)$, $\left.\min \left\{A_{e_{i}}^{+I}(x), B_{e_{i}}^{+I}(x)\right\} \geq\left(\lambda_{\mathrm{e}_{\mathrm{i}}}^{I} \vee \mu_{\mathrm{e}_{\mathrm{i}}}^{\mathrm{I}}\right)(\mathrm{X}), \min A_{e_{i}}^{+F}(x), B_{e_{i}}^{+F}(x)\right\} \geq\left(\lambda_{\mathrm{e}_{\mathrm{i}}}^{F} \vee \mu_{\mathrm{e}_{\mathrm{i}}}^{\mathrm{F}}\right)(x)$ for all $\mathrm{e}_{\mathrm{i}} \in \mathrm{M} \cap N$ and for all $\mathrm{x} \in \mathrm{X}$. Then $(\mathrm{P}, \mathrm{M}) \cap_{\mathrm{R}}(Q, N)$ is an INSCS.

Proof:

Let $(\mathrm{P}, \mathrm{M})=\left\{\mathrm{P}\left(\mathrm{e}_{\mathrm{i}}\right)=\left\{<\mathrm{x}, \mathrm{A}_{\mathrm{e}_{\mathrm{i}}}(\mathrm{x}), \lambda_{\mathrm{e}_{\mathrm{i}}}(\mathrm{x})>: \mathrm{x} \in \mathrm{X}\right\} \mathrm{e}_{\mathrm{i}} \in \mathrm{M}\right\}$ and $(\mathrm{Q}, \mathrm{N})=\left\{\mathrm{Q}\left(\mathrm{e}_{\mathrm{i}}\right)=\left\{<\mathrm{x}, \mathrm{B}_{\mathrm{e}_{\mathrm{i}}}(\mathrm{x}), \mu_{\mathrm{e}_{\mathrm{i}}}(\mathrm{x})>: \mathrm{x} \in \mathrm{X}\right\} \mathrm{e}_{\mathrm{i}} \in \mathrm{N}\right\}$. Then by definition of an INSCS we have $A_{e_{i}}^{-T}(x) \leq \lambda_{e_{i}}^{T}(x) \leq A_{e_{i}}^{+T}(x)$, $A_{e_{i}}^{-I}(x) \leq \lambda_{e_{i}}^{I}(x) \leq A_{e_{i}}^{+I}(x), \quad A_{e_{i}}^{-F}(x) \leq \lambda_{e_{i}}^{F}(x) \leq A_{e_{i}}^{+F}(x) \quad$ for all $\mathrm{e}_{\mathrm{i}} \in \mathrm{M}$ and for all $\mathrm{x} \in \mathrm{X}$. And $B_{e_{i}}^{-T}(x) \leq \mu_{e_{i}}^{T}(x) \leq B_{e_{i}}^{+T}(x), B_{e_{i}}^{-I}(x) \leq \mu_{e_{i}}^{I}(x) \leq B_{e_{i}}^{+I}(x)$, $B_{e_{i}}^{-F}(x) \leq \mu_{e_{i}}^{F}(x) \leq B_{e_{i}}^{+F}(x)$ for all $\mathrm{e}_{\mathrm{i}} \in \mathrm{N}$ and for all $\mathrm{x} \in \mathrm{X}$. This implies , 
$\min \left\{A_{e_{i}}^{-T}(x), B_{e_{i}}^{-T}(x)\right\} \leq\left(\lambda_{\mathrm{e}_{\mathrm{i}}}^{T} \vee \mu_{\mathrm{e}_{\mathrm{i}}}^{\mathrm{T}}\right)(x) \quad \min \left\{A_{e_{i}}^{-I}(x), B_{e_{i}}^{-I}(x)\right\} \leq\left(\lambda_{\mathrm{e}_{\mathrm{i}}}^{I} \vee \mu_{\mathrm{e}_{\mathrm{i}}}^{\mathrm{I}}\right)(x)$ $\min \left\{A_{e_{i}}^{-F}(x), B_{e_{i}}^{-F}(x)\right\} \leq\left(\lambda_{\mathrm{e}_{\mathrm{i}}}^{F} \vee \mu_{\mathrm{e}_{\mathrm{i}}}^{\mathrm{F}}\right)(x)$, for all $\mathrm{e}_{\mathrm{i}} \in \mathrm{M} \cap N$ and for all $\mathrm{x} \in \mathrm{X}$ Alsosince $(\mathrm{P}, \mathrm{M}) \cap_{\mathrm{R}}(Q, N)=(H, C)$ where $\mathrm{M} \cap N=C, H\left(e_{i}\right)=P\left(e_{i}\right) \wedge_{R}$ $Q\left(e_{i}\right)$ if $e \in M \cap N$ then $P\left(e_{i}\right) \wedge_{R} Q\left(e_{i}\right)$ is defined as $P\left(e_{i}\right) \wedge_{R} Q\left(e_{i}\right)=H\left(e_{i}\right)=\left\{<x, \min \left\{A_{e_{i}}(x), B_{e_{i}}(x)\right\},\left(\lambda_{e_{i}} \vee \mu_{e_{i}}\right)(x), x \in X\right.$, $\left.e_{i} \in M \cap N\right\}$ Given condition $\max \left\{A_{e_{i}}^{+T}(x), B_{e_{i}}^{+T}(x)\right\} \geq\left(\lambda_{\mathrm{e}_{\mathrm{i}}}^{T} \vee \mu_{\mathrm{e}_{\mathrm{i}}}^{\mathrm{T}}\right)(x)$, $\left.\max \left\{A_{e_{i}}^{+I}(x), B_{e_{i}}^{+I}(x)\right\} \geq\left(\lambda_{e_{i}}^{I} \vee \mu_{e_{i}}^{\mathrm{I}}\right)(x) \max \left\{A_{e_{i}}^{+F}(x), B_{e_{i}}^{+F}(x)\right\} \geq \lambda_{e_{\mathrm{i}}}^{F} \vee \mu_{e_{\mathrm{i}}}^{\mathrm{F}}\right)(x)$, for all $\mathrm{e}_{\mathrm{i}} \in \mathrm{M} \cap N$ and for all $\mathrm{x} \in \mathrm{X}$. Thus from given condition and definition of INSCS $\left.\min \left\{A_{e_{i}}^{-T}(x), B_{e_{i}}^{-T}(x)\right\} \leq \lambda_{e_{\mathrm{i}}}^{T} \vee \mu_{e_{\mathrm{i}}}^{\mathrm{T}}\right)(x) \leq \min \left\{A_{e_{i}}^{+T}(x)\right.$ $\left.\left.B_{e_{i}}^{+T}(x)\right\}, \min \left\{A_{e_{i}}^{-I}(x), B_{e_{i}}^{-I}(x)\right\} \leq \lambda_{\mathrm{e}_{\mathrm{i}}}^{I} \vee \mu_{\mathrm{e}_{\mathrm{i}}}^{\mathrm{I}}\right)(x) \leq \min \left\{A_{e_{i}}^{+I}(x), B_{e_{i}}^{+I}(x)\right\}$ $\left.\min \left\{A_{e_{i}}^{-F}(x), B_{e_{i}}^{-F}(x)\right\} \leq \lambda_{\mathrm{e}_{\mathrm{i}}}^{F} \vee \mu_{\mathrm{e}_{\mathrm{i}}}^{\mathrm{F}}\right)(x) \leq \min \left\{A_{e_{i}}^{+F}(x), B_{e_{i}}^{+F}(x)\right\} \quad$ for all $\mathrm{e}_{\mathrm{i}} \in$ $\mathrm{M} \cap N$ and for all $\mathrm{x} \in \mathrm{X}$. Hence $(\mathrm{P}, \mathrm{M}) \cap_{\mathrm{R}}(Q, N)$ is an INSCS.

\section{Example: 3.6}

Let $(P, I)$ and $(Q, J)$ be T-external neutrosophic soft cubic sets (T-ENSCS) in $\mathrm{X}$ where $(\mathrm{P}, \mathrm{I})=\mathrm{P}\left(\mathrm{e}_{1}\right)=\left\{<\mathrm{x},([0.2,0.5],[0.5,0.7],[0.3,0.5]),(0.7,0.6,0.8)>\mathrm{e}_{1} \in \mathrm{I}\right\}$ $(\mathrm{Q}, \mathrm{J})=\mathrm{Q}\left(\mathrm{e}_{1}\right)=\left\{<\mathrm{x},([0.6,0.8][0.6,0.7][0.7,0.9]),(0.9,0.7,0.3)>\mathrm{e}_{1} \in \mathrm{J}\right\}$

for all $\mathrm{x} \in \mathrm{X}$

Then $(P, I)$ and $(Q, J)$ areT-ENSCSinXand $(\mathrm{P}, \mathrm{I}) \cup_{R}(Q, J)=(P, I) \cup(Q, J)=$ $P \cup Q\left(e_{1}\right)=\left\{<\mathrm{x},([0.6,0.8][0.6,0.7],[0.7,0.9]),(0.7,0.6,0.3)>\mathrm{e}_{1} \in\right.$

$\mathrm{I} \cap \mathrm{J}\}$ for all $\mathrm{x} \in \mathrm{X}$.

$(\mathrm{P}, \mathrm{I}) \cup_{R}(Q, J)$ is not an T-ENSCS since

$$
\left(\lambda_{e_{1}}^{T} \wedge \mu_{e_{1}}^{T}\right)(x)=0.7 \in(0.6,0.8)=\left(\left(A_{e_{1}}^{T} \cup B_{e_{1}}^{T}\right)^{-}(x),\left(A_{e_{1}}^{T} \cup B_{e_{1}}^{T}\right)^{+}(x)\right)
$$


Cruz, RA

Irudayam, FN

From the above example it is clear that R-union of T-ENSCS may not be T-ENSCS. We provide a condition for the R- union of T-external (resp. I-external and F-external) neutrosophic soft cubic sets to be T-external (resp. I-external and F- external) neutrosophic soft cubic set.

\section{Theorem 3.7}

Let $(P, M)=\left\{P\left(e_{i}\right)=\left\{<x, A_{e_{i}}(x), \lambda_{e_{i}}(x)>: x \in X\right\} e_{i} \in M\right\}$ and $(\mathrm{Q}, \mathrm{N})=\left\{\mathrm{Q}\left(\mathrm{e}_{\mathrm{i}}\right)=\left\{<\mathrm{x}, \mathrm{B}_{\mathrm{e}_{\mathrm{i}}}(\mathrm{x}), \mu_{\mathrm{e}_{\mathrm{i}}}(\mathrm{x})>: \mathrm{x} \in \mathrm{X}\right\} \mathrm{e}_{\mathrm{i}} \in \mathrm{N}\right\}$ be T- ENSCSs in $\mathrm{X}$ such that

$$
\left(\lambda_{e_{i}}^{T} \wedge \mu_{e_{i}}^{T}\right)(x) \in\left(\begin{array}{l}
\max \left\{\left\{\min \left\{A_{e_{i}}^{+T}(x), B_{e_{i}}^{-T}(x)\right\}, \min \left\{A_{e_{i}}^{-T}(x), B_{e_{i}}^{+T}(x)\right\}\right\}\right. \\
\min \left\{\max \left\{A_{e_{i}}^{+T}(x), B_{e_{i}}^{-T}(x)\right\}, \max \left\{A_{e_{i}}^{-T}(x), B_{e_{i}}^{+T}(x)\right\}\right\}
\end{array}\right)
$$

for all $\mathrm{e}_{\mathrm{i}} \in \mathrm{M}$ and for all $\mathrm{e}_{\mathrm{i}} \in N$ and for all $\mathrm{x} \in \mathrm{X}$. Then $(\mathrm{P}, \mathrm{M}) \cup_{R}$ $(Q, N)$ is also an T- ENSCS.

Proof

Consider $(\mathrm{P}, \mathrm{M}) \cup_{R}(Q, N)=(H, C)$ where and $\mathrm{M} \cup N=C$

$$
H\left(e_{i}\right)=\left\{\begin{array}{ll}
P\left(e_{i}\right) & \text { if } e \in M-N \\
Q\left(e_{i}\right) & \text { if } e \in N-M \\
P\left(e_{i}\right) \vee_{R} Q\left(e_{i}\right) & \text { if } e \in M \cap N
\end{array}\right\}
$$

where $H\left(e_{i}\right)=P\left(e_{i}\right) \vee_{R} Q\left(e_{i}\right)$ is defined as

$P\left(e_{i}\right) \vee_{R} Q\left(e_{i}\right)=H\left(e_{i}\right)=\left\{<x, \max \left\{A_{e_{i}}(x), B_{e_{i}}(x)\right\},\left(\lambda_{e_{i}} \wedge \mu_{e_{i}}\right)(x), x \in X, e_{i} \in\right.$ $M \cap N\}$ where $P^{T}\left(e_{i}\right) \vee_{R} Q^{T}\left(e_{i}\right)=\left\{<x, \max \left\{A_{e_{i}}^{T}(x), B_{e_{i}}^{T}(x)\right\},\left(\lambda_{e_{i}}^{T} \wedge \mu_{e_{i}}^{T}\right)\right.$ $\left.(x), x \in X, e_{i} \in M \cap N\right\}$, If $e_{i} \in M \cap N$. Take $\alpha_{e_{i}}^{T}=\min \left\{\max \left\{A_{e_{i}}^{+T}(x)\right.\right.$, $\left.\left.B_{e_{i}}^{-T}(x)\right\}, \max \left\{A_{e_{i}}^{-T}(x), B_{e_{i}}^{+T}(x)\right\}\right\}$ and $\beta_{e_{i}}^{T}=\max \left\{\left\{\min \left\{A_{e_{i}}^{+T}(x), B_{e_{i}}^{-T}(x)\right\}\right.\right.$, 
$\left.\min \left\{A_{e_{i}}^{-T}(x), B_{e_{i}}^{+T}(x)\right\}\right\}$. Then $\alpha_{e_{i}}^{T}$ is one of $A_{e_{i}}^{-T}(x), B_{e_{i}}^{-T}(x), \alpha_{e_{i}}^{T} A_{e_{i}}^{+T}(x)$, $B_{e_{i}}^{+T}(x)$. Now we consider $\alpha_{e_{i}}^{T}=B_{e_{i}}^{-T}(x)$ or $B_{e_{i}}^{-T}(x)$ only as the remaining cases are similar to this one.

If $\alpha_{e_{i}}^{T}=B_{e_{i}}^{-T}(x)$ then $A_{e_{i}}^{-T}(x) \leq A_{e_{i}}^{+T}(x), \leq B_{e_{i}}^{-T}(x) \leq B_{e_{i}}^{+T}(x)$ and so $\beta_{e_{i}}^{T}=A_{e_{i}}^{+T}(x)$. Thus $\left(\mathrm{A}_{e_{i}}^{T} \cup B_{e_{i}}^{T}\right)^{-}(x)=B_{e_{i}}^{-T}(x)=\alpha_{e_{i}}^{T}>\left(\lambda_{e_{i}}^{T} \wedge \mu_{e_{i}}^{T}\right)(x)$. Hence $\left.\left(\lambda_{e_{i}}^{T} \wedge \mu_{e_{i}}^{T}\right)(x) \notin\left(\left(\mathrm{A}_{e_{i}}^{T} \cup B_{e_{i}}^{T}\right)^{-}(x), \mathrm{A}_{e_{i}}^{T} \cup B_{e_{i}}^{T}\right)^{+}(x)\right)$.

If $\quad \alpha_{e_{i}}^{T}=B_{e_{i}}^{+T}(x) \quad$ then $\quad A_{e_{i}}^{-T}(x) \leq B_{e_{i}}^{+T}(x) \leq A_{e_{i}}^{+T}(x), \quad$ and so $\beta_{e_{i}}^{T}=\max \left\{A_{e_{i}}^{-T}(x), B_{e_{i}}^{-T}(x)\right\}$. Assume that $\beta_{e_{i}}^{T}=A_{e_{i}}^{-T}(x)$ then we have $B_{e_{i}}^{-T}(x) \leq A_{e_{i}}^{-T}(x)<\left(\lambda_{e_{i}}^{T} \wedge \mu_{e_{i}}^{T}\right)(x) \leq B_{e_{i}}^{+T}(x) \leq A_{e_{i}}^{+T}(x)$. So from this we can write $\quad B_{e_{i}}^{-T}(x) \leq A_{e_{i}}^{-T}(x)<\left(\lambda_{e_{i}}^{T} \wedge \mu_{e_{i}}^{T}\right)(x)<B_{e_{i}}^{+T}(x) \leq A_{e_{i}}^{+T}(x)$. or $B_{e_{i}}^{-T}(x) \leq A_{e_{i}}^{-T}(x)=\left(\lambda_{e_{i}}^{T} \wedge \mu_{e_{i}}^{T}\right)(x)<B_{e_{i}}^{+T}(x) \leq A_{e_{i}}^{+T}(x)$. For this case $B_{e_{i}}^{-T}(x) \leq A_{e_{i}}^{-T}(x)<\left(\lambda_{e_{i}}^{T} \wedge \mu_{e_{i}}^{T}\right)(x)<B_{e_{i}}^{+T}(x) \leq A_{e_{i}}^{+T}(x)$ it is contradiction to the fact that and are T-ENSCS.

For the case $B_{e_{i}}^{-T}(x)<A_{e_{i}}^{-T}(x)=\left(\lambda_{e_{i}}^{T} \wedge \mu_{e_{i}}^{T}\right)(x) \leq B_{e_{i}}^{+T}(x) \leq A_{e_{i}}^{+T}(x)$ we have $\left.\left(\lambda_{e_{i}}^{T} \wedge \mu_{e_{i}}^{T}\right)(x) \notin\left(\left(\mathrm{A}_{e_{i}}^{T} \cup B_{e_{i}}^{T}\right)^{-}(x), \mathrm{A}_{e_{i}}^{T} \cup B_{e_{i}}^{T}\right)^{+}(x)\right) \quad$ because $\left(\mathrm{A}_{e_{i}}^{T} \cup B_{e_{i}}^{T}\right)^{-}(x)=A_{e_{i}}^{-T}(x)=\left(\lambda_{e_{i}}^{T} \wedge \mu_{e_{i}}^{T}\right)(x)$. Again assume that $\beta_{e_{i}}^{T}=B_{e_{i}}^{-T}(x)$ then we have $A_{e_{i}}^{-T}(x) \leq B_{e_{i}}^{-T}(x) \leq\left(\lambda_{e_{i}}^{T} \wedge \mu_{e_{i}}^{T}\right)(x) \leq B_{e_{i}}^{+T}(x) \leq A_{e_{i}}^{+T}(x)$.

From this we can write $A_{e_{i}}^{-T}(x) \leq B_{e_{i}}^{-T}(x)<\left(\lambda_{e_{i}}^{T} \wedge \mu_{e_{i}}^{T}\right)(x)<A_{e_{i}}^{+T}(x)$ $\leq B_{e_{i}}^{+T}(x)$ or $\quad A_{e_{i}}^{-T}(x) \leq B_{e_{i}}^{-T}(x)=\left(\lambda_{e_{i}}^{T} \wedge \mu_{e_{i}}^{T}\right)(x)<B_{e_{i}}^{+T}(x) \leq A_{e_{i}}^{+T}(x)$.
More on R-Union and R-Intersection of Neutrosophic Soft Cubic Set 
Cruz, RA Irudayam, FN

For this case $A_{e_{i}}^{-T}(x) \leq B_{e_{i}}^{-T}(x)<\left(\lambda_{e_{i}}^{T} \wedge \mu_{e_{i}}^{T}\right)(x)<B_{e_{i}}^{+T}(x) \leq A_{e_{i}}^{+T}(x)$ it is contradiction to the fact that and are T-ENSCS. And if we take the case $A_{e_{i}}^{-T}(x) \leq B_{e_{i}}^{-T}(x)=\left(\lambda_{e_{i}}^{T} \wedge \mu_{e_{i}}^{T}\right)(x) \leq A_{e_{i}}^{+T}(x) \leq B_{e_{i}}^{+T}(x)$, we get have $\left.\left(\lambda_{e_{i}}^{T} \wedge \mu_{e_{i}}^{T}\right)(x) \notin\left(\left(\mathrm{A}_{e_{i}}^{T} \cup B_{e_{i}}^{T}\right)^{-}(x), \mathrm{A}_{e_{i}}^{T} \cup B_{e_{i}}^{T}\right)^{+}(x)\right)$ because $\left(\mathrm{A}_{e_{i}}^{T} \cup B_{e_{i}}^{T}\right)^{-}(x)=$ $B_{e_{i}}^{-T}(x)=\left(\lambda_{e_{i}}^{T} \wedge \mu_{e_{i}}^{T}\right)(x)$.

If $\mathrm{e}_{\mathrm{i}} \in \mathrm{M}-N$ or $\mathrm{e}_{\mathrm{i}} \in N-M$, then result is trivial.

Hence $(\mathrm{P}, \mathrm{M}) \cup_{R}(Q, N)$ is T-ENSCS in $\mathrm{X}$.

Similarly we have the following theorems

\section{Theorem:3.8}

Let $(\mathrm{P}, \mathrm{M})=\left\{\mathrm{P}\left(\mathrm{e}_{\mathrm{i}}\right)=\left\{<\mathrm{x}, \mathrm{A}_{\mathrm{e}_{\mathrm{i}}}(\mathrm{x}), \lambda_{\mathrm{e}_{\mathrm{i}}}(\mathrm{x})>: \mathrm{x} \in \mathrm{X}\right\} \mathrm{e}_{\mathrm{i}} \in \mathrm{M}\right\}$ and $(\mathrm{Q}, \mathrm{N})=\left\{\mathrm{Q}\left(\mathrm{e}_{\mathrm{i}}\right)=\left\{<\mathrm{x}, \mathrm{B}_{\mathrm{e}_{\mathrm{i}}}(\mathrm{x}), \mu_{\mathrm{e}_{\mathrm{i}}}(\mathrm{x})>: \mathrm{x} \in \mathrm{X}\right\} \mathrm{e}_{\mathrm{i}} \in \mathrm{N}\right\}$ be I- ENSCSs in $\mathrm{X}$ such that

$$
\left(\lambda_{e_{i}}^{I} \vee \mu_{e_{i}}^{I}\right)(x) \in\left(\begin{array}{l}
\max \left\{\left\{\min \left\{A_{e_{i}}^{+I}(x), B_{e_{i}}^{-I}(x)\right\}, \min \left\{A_{e_{i}}^{-I}(x), B_{e_{i}}^{+I}(x)\right\}\right\}\right. \\
\min \left\{\max \left\{A_{e_{i}}^{+I}(x), B_{e_{i}}^{-I}(x)\right\}, \max \left\{A_{e_{i}}^{-I}(x), B_{e_{i}}^{+I}(x)\right\}\right\}
\end{array}\right)
$$

for all $\mathrm{e}_{\mathrm{i}} \in \mathrm{M}$ and for all $\mathrm{e}_{\mathrm{i}} \in \mathrm{N}$ and for all $\mathrm{x} \in \mathrm{X}$.Then $(\mathrm{P}, \mathrm{M}) \cup_{R}(Q, N)$ is also an I- ENSCS.

\section{Theorem 3.9}

Let $(\mathrm{P}, \mathrm{M})=\left\{\mathrm{P}\left(\mathrm{e}_{\mathrm{i}}\right)=\left\{<\mathrm{x}, \mathrm{A}_{\mathrm{e}_{\mathrm{i}}}(\mathrm{x}), \lambda_{\mathrm{e}_{\mathrm{i}}}(\mathrm{x})>: \mathrm{x} \in \mathrm{X}\right\} \mathrm{e}_{\mathrm{i}} \in \mathrm{M}\right\}$ and $(\mathrm{Q}, \mathrm{N})=\left\{\mathrm{Q}\left(\mathrm{e}_{\mathrm{i}}\right)=\left\{<\mathrm{x}, \mathrm{B}_{\mathrm{e}_{\mathrm{i}}}(\mathrm{x}), \mu_{\mathrm{e}_{\mathrm{i}}}(\mathrm{x})>: \mathrm{x} \in \mathrm{X}\right\} \mathrm{e}_{\mathrm{i}} \in \mathrm{N}\right\}$ be F- ENSCSs in $\mathrm{X}$ such that 
$\left(\lambda_{e_{i}}^{F} \vee \mu_{e_{i}}^{F}\right)(x) \in\left(\begin{array}{c}\max \left\{\left\{\min \left\{A_{e_{i}}^{+F}(x), B_{e_{i}}^{-F}(x)\right\}, \min \left\{A_{e_{i}}^{-F}(x), B_{e_{i}}^{+F}(x)\right\}\right\},\right. \\ \min \left\{\max \left\{A_{e_{i}}^{+F}(x), B_{e_{i}}^{-F}(x)\right\}, \max \left\{A_{e_{i}}^{-F}(x), B_{e_{i}}^{+F}(x)\right\}\right\}\end{array}\right)$

for all $\mathrm{e}_{\mathrm{i}} \in \mathrm{M}$ and for all $\mathrm{e}_{\mathrm{i}} \in \mathrm{N}$ and for all $\mathrm{x} \in \mathrm{X}$. Then $(\mathrm{P}, \mathrm{M}) \cup_{R}(Q, N)$ is also F- ENSCS.

\section{Corollary: $\mathbf{3 . 1 0}$}

Let $(\mathrm{P}, \mathrm{M})=\left\{\mathrm{P}\left(\mathrm{e}_{\mathrm{i}}\right)=\left\{<\mathrm{x}, \mathrm{A}_{\mathrm{e}_{\mathrm{i}}}(\mathrm{x}), \lambda_{\mathrm{e}_{\mathrm{i}}}(\mathrm{x})>: \mathrm{x} \in \mathrm{X}\right\} \mathrm{e}_{\mathrm{i}} \in \mathrm{M}\right\}$ and $(\mathrm{Q}, \mathrm{N})=\left\{\mathrm{Q}\left(\mathrm{e}_{\mathrm{i}}\right)=\left\{<\mathrm{x}, \mathrm{B}_{\mathrm{e}_{\mathrm{i}}}(\mathrm{x}), \mu_{\mathrm{e}_{\mathrm{i}}}(\mathrm{x})>: \mathrm{x} \in \mathrm{X}\right\} \mathrm{e}_{\mathrm{i}} \in \mathrm{N}\right\}$ be NSCSs in $\mathrm{X}$. Then R-union $(\mathrm{P}, \mathrm{M}) \cup_{R}(Q, N)$ is also an ENSCS in $\mathrm{X}$ when the conditions (3.7), (3.8)and (3.9) are valid.

\section{Example: 3.11}

Let $(P, I)$ and $(Q, J)$ be T-external neutrosophic soft cubic sets (T- ENSCS) in $\mathrm{X}$ where

$(\mathrm{P}, \mathrm{I})=\mathrm{P}\left(\mathrm{e}_{1}\right)=\left\{<\mathrm{x},([0.3,0.5],[0.2,0.5],[0.5,0.7]),(0.2,0.3,0.4)>\mathrm{e}_{1} \in \mathrm{I}\right\}$,

$(\mathrm{Q}, \mathrm{J})=\mathrm{Q}\left(\mathrm{e}_{1}\right)=\left\{<\mathrm{x},([0.7,0.9][0.6,0.8][0.4,0.7]),(0.4,0.7,0.3)>\mathrm{e}_{1} \in \mathrm{J}\right\}$

for all $\mathrm{x} \in \mathrm{X}$

Then $(P, I)$ and $(Q, J)$ are T-ENSCS in $\mathrm{X}$ and $(\mathrm{P}, \mathrm{I}) \cap_{R}(Q, J)=(P, I) \cap$ $(Q, J)=P \cap Q\left(e_{1}\right)=\{<\mathrm{x},([0.3,0.5][0.2,0.5],[0.4,0.7]),(0.4,0.7,0.4)$

$\left.>\mathrm{e}_{1} \in \mathrm{I} \cap \mathrm{J}\right\}$ for all $\mathrm{x} \in \mathrm{X}$.

$(\mathrm{P}, \mathrm{I}) \cap_{R}(Q, J)$ is not T-ENSCS since

$$
\left(\lambda_{e_{1}}^{T} \vee \mu_{e_{2}}^{T}\right)(x)=0.4 \in(0.3 .0 .5)=\left(\left(A_{e_{1}}^{T} \cap B_{e_{1}}^{T}\right)^{-}(x),\left(A_{e_{1}}^{T} \cap B_{e_{1}}^{T}\right)^{+}(x)\right)
$$

From the above example it is clear that R-intersection of T-ENSCS may not be an T- ENSCS. We provide a condition for the R-intersection of T-external (resp. I-external and F-external) neutrosophic soft cubic sets to be T-external (resp. I-external and F-external) neutrosophic soft cubic set.

\section{Theorem 3.12}

Let $(P, M)=\left\{P\left(e_{i}\right)=\left\{<x, A_{e_{i}}(x), \lambda_{e_{i}}(x)>: x \in X\right\} e_{i} \in M\right\}$ and 
Cruz, RA Irudayam, FN

$(\mathrm{Q}, \mathrm{N})=\left\{\mathrm{Q}\left(\mathrm{e}_{\mathrm{i}}\right)=\left\{<\mathrm{x}, \mathrm{B}_{\mathrm{e}_{\mathrm{i}}}(\mathrm{x}), \mu_{\mathrm{e}_{\mathrm{i}}}(\mathrm{x})>: \mathrm{x} \in \mathrm{X}\right\} \mathrm{e}_{\mathrm{i}} \in \mathrm{N}\right\}$ be $\mathrm{T}-$ ENSCSs in $\mathrm{X}$ such that

$$
H\left(e_{i}\right)=\left\{\begin{array}{ll}
P\left(e_{i}\right) & \text { if } e \in M-N \\
Q\left(e_{i}\right) & \text { if } e \in N-M \\
P\left(e_{i}\right) \wedge_{R} Q\left(e_{i}\right) & \text { if } e \in M \cap N
\end{array}\right\}
$$

for all $\mathrm{e}_{\mathrm{i}} \in \mathrm{M}$ and for all $\mathrm{e}_{\mathrm{i}} \in \mathrm{N}$ and for all $\mathrm{x} \in \mathrm{X}$. Then $(\mathrm{P}, \mathrm{M}) \cap_{R}(Q, N)$ is also an T- ENSCS.

Proof:

Consider $(\mathrm{P}, \mathrm{M}) \cap_{R}(Q, N)=(H, C)$ where $\mathrm{I} \cap J=C$ and

$$
H\left(e_{i}\right)=\left\{\begin{array}{ll}
P\left(e_{i}\right) & \text { if } e \in M-N \\
Q\left(e_{i}\right) & \text { if } e \in N-M \\
P\left(e_{i}\right) \wedge_{R} Q\left(e_{i}\right) & \text { if } e \in M \cap N
\end{array}\right\}
$$

where $H\left(e_{i}\right)=P\left(e_{i}\right) \wedge_{R} Q\left(e_{i}\right)$ is defined as

$P\left(e_{i}\right) \wedge_{R} Q\left(e_{i}\right)=H\left(e_{i}\right)=\left\{<x, \min \left\{A_{e_{i}}(x), B_{e_{i}}(x)\right\},\left(\lambda_{e_{i}} \vee \mu_{e_{i}}\right)(x), x \in X\right.$, $\left.e_{i} \in M \cap N\right\}$, where For each $e_{i} \in M \cap N$, Take $\alpha_{e_{i}}^{T}=\min \left\{\max \left\{A_{e_{i}}^{+T}\right.\right.$ $\left.\left.(x), B_{e_{i}}^{-T}(x)\right\}, \max \left\{A_{e_{i}}^{-T}(x), B_{e_{i}}^{+T}(x)\right\}\right\}$ and $\beta_{e_{i}}^{T}=\max \left\{\left\{\min \left\{A_{e_{i}}^{+T}(x), B_{e_{i}}^{-T}\right.\right.\right.$ $\left.\left.{ }^{\prime}(x)\right\}, \min \left\{A_{e_{i}}^{-T}(x), B_{e_{i}}^{+T}(x)\right\}\right\}$. Then $\alpha_{e_{i}}^{T}$ is one of $A_{e_{i}}^{-T}(x), B_{e_{i}}^{-T}(x), A_{e_{i}}^{+T}(x)$ and $B_{e}^{+T}(x)$. Now we consider $\alpha_{e_{i}}^{T}=B_{e_{i}}^{-T}(x)$ or $B_{e_{i}}^{-T}(x)$ only as the
remaining cases are similar to this one. If $\alpha_{e_{i}}^{T}=B_{e_{i}}^{-T}(x)$ then $A_{e_{i}}^{-T}(x) \leq A_{e_{i}}^{+T}(x), \leq B_{e_{i}}^{-T}(x) \leq B_{e_{i}}^{+T}(x)$ and so $\beta_{e_{i}}^{T}=A_{e_{i}}^{+T}(x)$.Then given inequality we have $\left(\mathrm{A}_{e_{i}}^{T} \cap B_{e_{i}}^{T}\right)^{+}(x)=A_{e_{i}}^{+T}(x)=\beta_{e_{i}}^{T}$ $<\left(\lambda_{e_{i}}^{T} \vee \mu_{e_{i}}^{T}\right)(x)$. Thus we have $\left(\lambda_{e_{i}}^{T} \vee \mu_{e_{i}}^{T}\right)(x) \notin\left(\left(\mathrm{A}_{e_{i}}^{T} \cup B_{e_{i}}^{T}\right)^{-}(x), \mathrm{A}_{e_{i}}^{T} \cup B_{e_{i}}^{T}\right)^{+}$ $(x))$.

If $\alpha_{e_{i}}^{T}=B_{e_{i}}^{+T}(x)$ then $A_{e_{i}}^{-T}(x) \leq B_{e_{i}}^{+T}(x) \leq A_{e_{i}}^{+T}(x)$, and so $\beta_{e_{i}}^{T}=\max \left\{A_{e_{i}}^{-T}(x), B_{e_{i}}^{-T}(x)\right\}$. Assume that $\beta_{e_{i}}^{T}=A_{e_{i}}^{-T}(x)$ 
then we have $B_{e_{i}}^{-T}(x) \leq A_{e_{i}}^{-T}(x)<\left(\lambda_{e_{i}}^{T} \vee \mu_{e_{i}}^{T}\right)(x)<B_{e_{i}}^{+T}(x) \leq A_{e_{i}}^{+T}(x)$. So from this we can write $B_{e_{i}}^{-T}(x) \leq A_{e_{i}}^{-T}(x)<\left(\lambda_{e_{i}}^{T} \vee \mu_{e_{i}}^{T}\right)(x)=B_{e_{i}}^{+T}$

$(x) \leq A_{e_{i}}^{+T}(x)$ or $\quad B_{e_{i}}^{-T}(x) \leq A_{e_{i}}^{-T}(x)<\left(\lambda_{e_{i}}^{T} \vee \mu_{e_{i}}^{T}\right)(x)<B_{e_{i}}^{+T}(x) \leq A_{e_{i}}^{+T}(x)$. For this case $B_{e_{i}}^{-T}(x)<A_{e_{i}}^{-T}(x)=\left(\lambda_{e_{i}}^{T} \vee \mu_{e_{i}}^{T}\right)(x) \leq B_{e_{i}}^{+T}(x) \leq A_{e_{i}}^{+T}(x)$ it is contradiction to the fact that and (P,M) are (Q.N) T-ENSCS.

For the case $B_{e_{i}}^{-T}(x)<A_{e_{i}}^{-T}(x)=\left(\lambda_{e_{i}}^{T} \vee \mu_{e_{i}}^{T}\right)(x) \leq B_{e_{i}}^{+T}(x) \leq A_{e_{i}}^{+T}(x)$ we have $\left.\left(\lambda_{e_{i}}^{T} \vee \mu_{e_{i}}^{T}\right)^{e_{i}}(x) \notin\left(\left(\mathrm{A}_{e_{i}}^{T} \cup B_{e_{i}}^{T}\right)^{-}(x), \mathrm{A}_{e_{i}}^{T} \cup B_{e_{i}}^{T}\right)^{+}(x)\right)$ because $\left(\mathrm{A}_{e_{i}}^{T} \cup B_{e_{i}}^{T}\right)^{+}$ $(x)=B_{e_{i}}^{-T}(x)=\left(\lambda_{e_{i}}^{T} \vee \mu_{e_{i}}^{T}\right)(x)$. Again assume that $\beta_{e_{i}}^{T}=B_{e_{i}}^{-T}(x)$ then we have $A_{e_{i}}^{-T}(x) \leq B_{e_{i}}^{-T}(x) \leq\left(\lambda_{e_{i}}^{T} \vee \mu_{e_{i}}^{T}\right)(x) \leq B_{e_{i}}^{+T}(x) \leq A_{e_{i}}^{+T}(x)$. From this we can write $A_{e_{i}}^{-T}(x) \leq B_{e_{i}}^{-T}(x)<\left(\lambda_{e_{i}}^{T} \vee \mu_{e_{i}}^{T}\right)(x)<B_{e_{i}}^{+T}(x) \leq A_{e_{i}}^{+T}(x)$ or $A_{e_{i}}^{-T}(x) \leq B_{e_{i}}^{-T}(x)<\left(\lambda_{e_{i}}^{T} \vee \mu_{e_{i}}^{T}\right)(x)=B_{e_{i}}^{+T}(x) \leq A_{e_{i}}^{+T}(x)$. For the case $A_{e_{i}}^{-T}(x) \leq B_{e_{i}}^{-T}(x)<\left(\lambda_{e_{i}}^{T} \vee \mu_{e_{i}}^{T}\right)(x)<B_{e_{i}}^{+T}(x) \leq A_{e_{i}}^{+T}(x)$ it is

contradiction to the fact that and are T-ENSCS. And if we take the case $A_{e_{i}}^{-T}(x) \leq B_{e_{i}}^{-T}(x)<\left(\lambda_{e_{i}}^{T} \vee \mu_{e_{i}}^{T}\right)(x)=B_{e_{i}}^{+T}(x) \leq A_{e_{i}}^{+T}(x)$, we get have $\left.\left(\begin{array}{c}\lambda_{e_{i}}^{T} \vee \mu_{e_{i}}^{T} \\ e^{\prime}\end{array}\right)(x) \notin\left(\left(\mathrm{A}_{e_{i}}^{T} \cup B_{e_{i}}^{T}\right)^{-}(x), \mathrm{A}_{e_{i}}^{T} \cup B_{e_{i}}^{T}\right)^{+}(x)\right)$ because $\left(\mathrm{A}_{e_{i}}^{T} \cup B_{e_{i}}^{T}\right)^{+}(x)=$ $B_{e_{i}}^{+T}(x)=\left(\lambda_{e_{i}}^{T} \vee \mu_{e_{i}}^{T}\right)(x)$. Hence $(\mathrm{P}, \mathrm{M}) \cap_{R}(Q, N)$ is T-ENSCS in $\mathrm{X}$ for $e_{i} \in M \cap N$.

Similarly we have the following theorems.

\section{Theorem 3.12}

Let $(P, M)=\left\{P\left(e_{i}\right)=\left\{<x, A_{e_{i}}(x), \lambda_{e_{i}}(x)>: x \in X\right\} e_{i} \in M\right\}$ and $(\mathrm{Q}, \mathrm{N})=\left\{\mathrm{Q}\left(\mathrm{e}_{\mathrm{i}}\right)=\left\{<\mathrm{x}, \mathrm{B}_{\mathrm{e}_{\mathrm{i}}}(\mathrm{x}), \mu_{\mathrm{e}_{\mathrm{i}}}(\mathrm{x})>: \mathrm{x} \in \mathrm{X}\right\} \mathrm{e}_{\mathrm{i}} \in \mathrm{N}\right\}$ be I- ENSCSs in $\mathrm{X}$ such that
More on R-Union and R-Intersection of Neutrosophic Soft Cubic Set 
Cruz, RA

Irudayam, FN

$$
\left(\lambda_{e_{i}}^{I} \vee \mu_{e_{i}}^{I}\right)(x) \in\left(\begin{array}{l}
\max \left\{\left\{\min \left\{A_{e_{i}}^{+I}(x), B_{e_{i}}^{-I}(x)\right\}, \min \left\{A_{e_{i}}^{-I}(x), B_{e_{i}}^{+I}(x)\right\}\right\}\right. \\
\min \left\{\max \left\{A_{e_{i}}^{+I}(x), B_{e_{i}}^{-I}(x)\right\}, \max \left\{A_{e_{i}}^{-I}(x), B_{e_{i}}^{+I}(x)\right\}\right\}
\end{array}\right)
$$

for all $\mathrm{e}_{\mathrm{i}} \in \mathrm{M}$ and for all $\mathrm{e}_{\mathrm{i}} \in$ Nand for all $\mathrm{x} \in \mathrm{X}$. Then $(\mathrm{P}, \mathrm{M}) \cap_{R}(Q, N)$ is also an I- ENSCS.

\section{Theorem 3.13}

Let $(P, M)=\left\{P\left(e_{i}\right)=\left\{<x, A_{e_{i}}(x), \lambda_{e_{i}}(x)>: x \in X\right\} e_{i} \in M\right\}$ and

$(\mathrm{Q}, \mathrm{N})=\left\{\mathrm{Q}\left(\mathrm{e}_{\mathrm{i}}\right)=\left\{<\mathrm{x}, \mathrm{B}_{\mathrm{e}_{\mathrm{i}}}(\mathrm{x}), \mu_{\mathrm{e}_{\mathrm{i}}}(\mathrm{x})>: \mathrm{x} \in \mathrm{X}\right\} \mathrm{e}_{\mathrm{i}} \in \mathrm{N}\right\}$ be F- ENSCSs in $\mathrm{X}$ such that

$$
\left(\lambda_{e_{i}}^{F} \vee \mu_{e_{i}}^{F}\right)(x) \in\left(\begin{array}{c}
\max \left\{\left\{\min \left\{A_{e_{i}}^{+F}(x), B_{e_{i}}^{-F}(x)\right\}, \min \left\{A_{e_{i}}^{-F}(x), B_{e_{i}}^{+F}(x)\right\}\right\}\right. \\
\min \left\{\max \left\{A_{e_{i}}^{+F}(x), B_{e_{i}}^{-F}(x)\right\}, \max \left\{A_{e_{i}}^{-F}(x), B_{e_{i}}^{+F}(x)\right\}\right\}
\end{array}\right)
$$

for all $\mathrm{e}_{\mathrm{i}} \in \mathrm{M}$ and for all $\mathrm{e}_{\mathrm{i}} \in \mathrm{N}$ and for all $\mathrm{x} \in \mathrm{X}$. Then $(\mathrm{P}, \mathrm{M}) \cap_{R}(Q, N)$ is also F- ENSCS.

\section{Corollary:3.14}

Let $(\mathrm{P}, \mathrm{M})=\left\{\mathrm{P}\left(\mathrm{e}_{\mathrm{i}}\right)=\left\{<\mathrm{x}, \mathrm{A}_{\mathrm{e}_{\mathrm{i}}}(\mathrm{x}), \lambda_{\mathrm{e}_{\mathrm{i}}}(\mathrm{x})>: \mathrm{x} \in \mathrm{X}\right\} \mathrm{e}_{\mathrm{i}} \in \mathrm{M}\right\}$ and $(\mathrm{Q}, \mathrm{N})=\left\{\mathrm{Q}\left(\mathrm{e}_{\mathrm{i}}\right)=\left\{<\mathrm{x}, \mathrm{B}_{\mathrm{e}_{\mathrm{i}}}(\mathrm{x}), \mu_{\mathrm{e}_{\mathrm{i}}}(\mathrm{x})>: \mathrm{x} \in \mathrm{X}\right\} \mathrm{e}_{\mathrm{i}} \in \mathrm{N}\right\}$ be NSCSs in $\mathrm{X}$.

Then $(\mathrm{P}, \mathrm{M}) \cap_{R}(Q, N)$ is also an ENSCS in $\mathrm{X}$ when the conditions (3.11), (3.12) and (3.13) are valid.

\section{Theorem 3.15}

Let $(\mathrm{P}, \mathrm{M})=\left\{\mathrm{P}\left(\mathrm{e}_{\mathrm{i}}\right)=\left\{<\mathrm{x}, \mathrm{A}_{\mathrm{e}_{\mathrm{i}}}(\mathrm{x}), \lambda_{\mathrm{e}_{\mathrm{i}}}(\mathrm{x})>: \mathrm{x} \in \mathrm{X}\right\} \mathrm{e}_{\mathrm{i}} \in \mathrm{M}\right\}$ and $(\mathrm{Q}, \mathrm{N})=\left\{\mathrm{Q}\left(\mathrm{e}_{\mathrm{i}}\right)=\left\{<\mathrm{x}, \mathrm{B}_{\mathrm{e}_{\mathrm{i}}}(\mathrm{x}), \mu_{\mathrm{e}_{\mathrm{i}}}(\mathrm{x})>: \mathrm{x} \in \mathrm{X}\right\} \mathrm{e}_{\mathrm{i}} \in \mathrm{N}\right\}$ be T- external neutrosophic soft cubic sets in $\mathrm{X}$ such that $\min \left\{\max \left\{A_{e_{i}}^{+T}(x), B_{e_{i}}^{-T}(x)\right\}\right.$, max $\left.\left\{A_{e_{i}}^{-T}(x), B_{e_{i}}^{+T}(x)\right\}\right\}=\left(\lambda_{e_{i}}^{T} \wedge \mu_{e_{i}}^{T}\right)(x)$ 


$$
=\max \left\{\left\{\min \left\{A_{e_{i}}^{+T}(x), B_{e_{i}}^{-T}(x)\right\}, \min \left\{A_{e_{i}}^{-T}(x), B_{e_{i}}^{+T}(x)\right\}\right\}\right.
$$

then the $(\mathrm{P}, \mathrm{M}) \cap_{R}(Q, N)$ is both an T-internal neutrosophic soft cubic set and an T-external neutrosophic soft cubic set in $\mathrm{X}$.

Proof: Consider $(\mathrm{P}, \mathrm{M}) \cap_{R}(Q, N)=(H, C)$ where $\quad \mathrm{M} \cap N=C$ where $H\left(e_{i}\right)=P\left(e_{i}\right) \wedge_{R} Q\left(e_{i}\right)$ is defined as $P\left(e_{i}\right) \wedge_{R} Q\left(e_{i}\right)=H\left(e_{i}\right)$ $\left.=\left\{<\mathrm{x}, \min \left\{\mathrm{A}_{\mathrm{e}_{\mathrm{i}}} \cdot(\mathrm{x}), \mathrm{B}_{\mathrm{e}_{\mathrm{i}}}(\mathrm{x})\right\},\left(\lambda_{\mathrm{e}_{\mathrm{i}}} \vee \mu_{\mathrm{e}_{\mathrm{i}}}\right)(\mathrm{x})>: \mathrm{x} \in \mathrm{X}\right\} \mathrm{e}_{\mathrm{i}} \in \mathrm{M} \cap \mathrm{N}\right\}$ Where $P^{T}\left(e_{i}\right) \wedge_{R} Q^{T}\left(e_{i}\right)=\left\{<\mathrm{x}, \min \left\{\mathrm{A}_{\mathrm{e}_{\mathrm{i}}}^{\mathrm{T}}(\mathrm{x}), \mathrm{B}_{\mathrm{e}_{\mathrm{i}}}^{\mathrm{T}}(\mathrm{x})\right\},\left(\lambda_{\mathrm{e}_{\mathrm{i}}}^{\mathrm{T}} \vee \mu_{\mathrm{e}_{\mathrm{i}}}^{\mathrm{T}}\right)(\mathrm{x})>: \mathrm{x} \in \mathrm{X}\right\}$ $\left.\mathrm{e}_{\mathrm{i}} \in \mathrm{M} \cap \mathrm{N}\right\}$. For each $\mathrm{e}_{\mathrm{i}} \in \mathrm{M} \cap \mathrm{N}$ Take $\alpha_{e_{i}}^{T}=\min \left\{\max \left\{A_{e_{i}}^{+T}(x), B_{e_{i}}^{-T}\right.\right.$ $\left.(x)\}, \max \left\{A_{e_{i}}^{-T}(x), B_{e_{i}}^{+T}(x)\right\}\right\}$ and $\beta_{e_{i}}^{T}=\max \left\{\left\{\min \left\{A_{e_{i}}^{+T}(x), B_{e_{i}}^{-T}(x)\right\}, \min \right.\right.$ $\left.\left\{A_{e_{i}}^{-T}(x), B_{e_{i}}^{+T}(x)\right\}\right\}$. Then $\alpha_{e_{i}}^{T}$ is one of $A_{e_{i}}^{-T}(x), B_{e_{i}}^{-T}(x), A_{e_{i}}^{+T}(x), B_{e_{i}}^{+T}(x)$. Now we consider $\alpha_{e_{i}}^{T}=A_{e_{i}}^{-T}(x)$, or $A_{e_{i}}^{+T}(x)$, only as the remaining cases are similar to this one. If $\alpha_{e_{i}}^{T}=A_{e_{i}}^{-T}(x)$ then $B_{e_{i}}^{-T}(x) \leq B_{e_{i}}^{+T}(x) \leq A_{e_{i}}^{-T}(x) \leq$ $A_{e_{i}}^{+T}(x)$, and so $\beta_{e_{i}}^{T}=B_{e_{i}}^{+T}(x)$. This implies that $A_{e_{i}}^{-T}(x)=\alpha_{e_{i}}^{T}=\left(\lambda_{e_{i}}^{T} \vee \mu_{e_{i}}^{T}\right)$ $(x)=\beta_{e_{i}}^{T}=B_{e_{i}}^{+T}(x)$. Thus $B_{e_{i}}^{-T}(x) \leq B_{e_{i}}^{+T}(x)=\left(\lambda_{e_{i}}^{T} \vee \mu_{e_{i}}^{T}\right)(x)=A_{e_{i}}^{-T}$ $(x) \leq A_{e_{j}}^{+T}(x)$. Which implies that $\left(\lambda_{e_{i}}^{T} \vee \mu_{e_{i}}^{T}\right)(x)=B_{e_{i}}^{+T}(x)=\left(\mathrm{A}_{e_{i}}^{T} \cap B_{e_{i}}^{T}\right)^{+}(x)$. Hence $\left(\lambda_{e_{i}}^{T} \vee \mu_{e_{i}}^{T}\right)(x) \notin\left(\left(\mathrm{A}_{e_{i}}^{T} \cap B_{e_{i}}^{T}\right)^{-}(x),\left(\mathrm{A}_{e_{i}}^{T} \cap B_{e_{i}}^{T}\right)^{+}(x)\right)$ and $\left(\mathrm{A}_{e_{i}}^{T} \cap B_{e_{i}}^{T}\right)^{-}(x)$ $\leq\left(\lambda_{e_{i}}^{T} \vee \mu_{e_{i}}^{T}\right)(x) \leq\left(\mathrm{A}_{e_{i}}^{T} \cap B_{e_{i}}^{T}\right)^{+}(x)$. If $\alpha_{e_{i}}^{T}=A_{e_{i}}^{+T}(x)$ then $B_{e_{i}}^{-T}(x) \leq A_{e_{i}}^{+T}$ $(x) \leq B_{e_{i}}^{+T}(x)$, and so $\left(\lambda_{e_{i}}^{T} \vee \mu_{e_{i}}^{T}\right)(x)=A_{e_{i}}^{+T}(x)=\left(\mathrm{A}_{e_{i}}^{T} \cap B_{e_{i}}^{T}\right)^{+}(x)$. Hence $\left(\lambda_{e_{i}}^{T} \vee \mu_{e_{i}}^{T}\right)(x) \notin\left(\left(\mathrm{A}_{e_{i}}^{T} \cap B_{e_{i}}^{T}\right)^{-}(x),\left(\mathrm{A}_{e_{i}}^{T} \cap B_{e_{i}}^{T}\right)^{+}(x)\right)$ and $\left(\mathrm{A}_{e_{i}}^{T} \cap B_{e_{i}}^{T}\right)^{-}(x) \leq\left(\lambda_{e_{i}}^{T}\right.$ $\left.\vee \mu_{e_{i}}^{T}\right)(x) \leq\left(\mathrm{A}_{e_{i}}^{T} \cap B_{e_{i}}^{T}\right)^{+}(x)$. Consequently we note that $(\mathrm{P}, \mathrm{M}) \cap_{R}(Q, N)$ is both an T-INSCS and an T-ENSCS in X.
More on R-Union and R-Intersection of Neutrosophic Soft Cubic Set 
Cruz, RA

Irudayam, FN

Similarly we have the following theorems

\section{Theorem 3.16}

If neutrosophic soft cubic set $(\mathrm{P}, \mathrm{M})=\left\{\mathrm{P}\left(\mathrm{e}_{\mathrm{i}}\right)=\left\{<\mathrm{x}, \mathrm{A}_{\mathrm{e}_{\mathrm{i}}}(\mathrm{x}), \lambda_{\mathrm{e}_{\mathrm{i}}}(\mathrm{x})>: \mathrm{x} \in\right.\right.$ $\left.\mathrm{X}\} \mathrm{e}_{\mathrm{i}} \in \mathrm{M}\right\}$ and $(\mathrm{Q}, \mathrm{N})=\left\{\mathrm{Q}\left(\mathrm{e}_{\mathrm{i}}\right)=\left\{<\mathrm{x}, \mathrm{B}_{\mathrm{e}_{\mathrm{i}}}(\mathrm{x}), \mu_{\mathrm{e}_{\mathrm{i}}}(\mathrm{x})>: \mathrm{x} \in \mathrm{X}\right\} \mathrm{e}_{\mathrm{i}} \in \mathrm{N}\right\}$ in X satisfy the following condition $\min \left\{\max \left\{A_{e_{i}}^{+I}(x), B_{e_{i}}^{-I}(x)\right\}, \max \left\{A_{e_{i}}^{-I}\right.\right.$

$$
\begin{aligned}
\left.\left.(x), B_{e_{i}}^{+I}(x)\right\}\right\} & =\left(\lambda_{e_{i}}^{I} \wedge \mu_{e_{i}}^{I}\right)(x) \\
= & \max \left\{\left\{\min \left\{A_{e_{i}}^{+I}(x), B_{e_{i}}^{-I}(x)\right\}, \min \left\{A_{e_{i}}^{-I}(x), B_{e_{i}}^{+I}(x)\right\}\right\}\right.
\end{aligned}
$$

then the $(\mathrm{P}, \mathrm{M}) \cap_{R}(Q, N)$ is both an I-INSCS and an I-ENSCS in X.

\section{Theorem 3.17}

If neutrosophic soft cubic set $(\mathrm{P}, \mathrm{M})=\left\{\mathrm{P}\left(\mathrm{e}_{\mathrm{i}}\right)=\left\{<\mathrm{x}, \mathrm{A}_{\mathrm{e}_{\mathrm{i}}}(\mathrm{x}), \lambda_{\mathrm{e}_{\mathrm{i}}}(\mathrm{x})>\right.\right.$ :

$\left.\mathrm{x} \in \mathrm{X}\} \mathrm{e}_{\mathrm{i}} \in \mathrm{M}\right\}$ and $(\mathrm{Q}, \mathrm{N})=\left\{\mathrm{Q}\left(\mathrm{e}_{\mathrm{i}}\right)=\left\{<\mathrm{x}, \mathrm{B}_{\mathrm{e}_{\mathrm{i}}}(\mathrm{x}), \mu_{\mathrm{e}_{\mathrm{i}}}(\mathrm{x})>: \mathrm{x} \in \mathrm{X}\right\} \mathrm{e}_{\mathrm{i}} \in \mathrm{N}\right\}$

in X satisfy the following condition $\min \left\{\max \left\{A_{e_{i}}^{+F}(x), B_{e_{i}}^{-F}(x)\right\}, \max \left\{A_{e_{i}}^{-F}\right.\right.$

$$
\begin{aligned}
&\left.\left.(x), B_{e_{i}}^{+F}(x)\right\}\right\}=\left(\lambda_{e_{i}}^{F} \wedge \mu_{e_{i}}^{F}\right)(x) \\
&=\max \left\{\left\{\min \left\{A_{e_{i}}^{+F}(x), B_{e_{i}}^{-F}(x)\right\}, \min \left\{A_{e_{i}}^{-F}(x), B_{e_{i}}^{+F}(x)\right\}\right\}\right.
\end{aligned}
$$

then the $(\mathrm{P}, \mathrm{M}) \cap_{R}(Q, N)$ is both an F-INSCS and an F-ENSCS in X.

\section{Corollary: 3.18}

Let $(P, M)=\left\{P\left(e_{i}\right)=\left\{<x, A_{e_{i}}(x), \lambda_{e_{i}}(x)>: x \in X\right\} e_{i} \in M\right\}$ and $(\mathrm{Q}, \mathrm{N})=\left\{\mathrm{Q}\left(\mathrm{e}_{\mathrm{i}}\right)=\left\{<\mathrm{x}, \mathrm{B}_{\mathrm{e}_{\mathrm{i}}}(\mathrm{x}), \mu_{\mathrm{e}_{\mathrm{i}}}(\mathrm{x})>: \mathrm{x} \in \mathrm{X}\right\} \mathrm{e}_{\mathrm{i}} \in \mathrm{N}\right\} \quad$ be NSCSs in $\mathrm{X}$. Then $(\mathrm{P}, \mathrm{M}) \cap_{R}(Q, N)$ is also an ENSCS and INSCS in $\mathrm{X}$ when the conditions (3.15), (3.16) and (3.17) are valid.

\section{Theorem: 3.19}

Let $(P, M)=\left\{P\left(e_{i}\right)=\left\{<x, A_{e_{i}}(x), \lambda_{e_{i}}(x)>: x \in X\right\} e_{i} \in M\right\}$ and 
$(\mathrm{Q}, \mathrm{N})=\left\{\mathrm{Q}\left(\mathrm{e}_{\mathrm{i}}\right)=\left\{<\mathrm{x}, \mathrm{B}_{\mathrm{e}_{\mathrm{i}}}(\mathrm{x}), \mu_{\mathrm{e}_{\mathrm{i}}}(\mathrm{x})>: \mathrm{x} \in \mathrm{X}\right\} \mathrm{e}_{\mathrm{i}} \in \mathrm{N}\right\}$ be T- INSCSs in X such that $\left(\lambda_{e_{i}}^{T} \wedge \mu_{e_{i}}^{T}\right)(x) \leq \max \left\{A_{e_{i}}^{-T}(x), B_{e_{i}}^{-T}(x)\right\}$ for all $\mathrm{e}_{\mathrm{i}} \in \mathrm{M}$ and for all $\mathrm{e}_{\mathrm{i}} \in N$ and for all $\mathrm{x} \in \mathrm{X}$, then $(\mathrm{P}, \mathrm{M}) \cup_{R}(Q, N)$ is an T-ENSCS in $\mathrm{X}$.

Proof:

Let $(\mathrm{P}, \mathrm{M})=\left\{\mathrm{P}\left(\mathrm{e}_{\mathrm{i}}\right)=\left\{<\mathrm{x}, \mathrm{A}_{\mathrm{e}_{\mathrm{i}}}(\mathrm{x}), \lambda_{\mathrm{e}_{\mathrm{i}}}(\mathrm{x})>: \mathrm{x} \in \mathrm{X}\right\} \mathrm{e}_{\mathrm{i}} \in \mathrm{M}\right\}$ and $(\mathrm{Q}, \mathrm{N})=\left\{\mathrm{Q}\left(\mathrm{e}_{\mathrm{i}}\right)=\left\{<\mathrm{x}, \mathrm{B}_{\mathrm{e}_{\mathrm{i}}}(\mathrm{x}), \mu_{\mathrm{e}_{\mathrm{i}}}(\mathrm{x})>: \mathrm{x} \in \mathrm{X}\right\} \mathrm{e}_{\mathrm{i}} \in \mathrm{N}\right\}$ are T- INSCSs in $\mathrm{X}$.

Thus for all $\mathrm{e}_{\mathrm{i}} \in \mathrm{M}$, we have $A_{e_{i}}^{-T}(x) \leq \lambda_{e_{i}}^{T}(x) \leq A_{e_{i}}^{+T}(x)$ and for all $\mathrm{e}_{\mathrm{i}} \in N$ $B_{e_{i}}^{-T}(x) \leq \mu_{e_{i}}^{T}(x) \leq B_{e_{i}}^{+T}(x)$. Since $(\mathrm{P}, \mathrm{M}) \cup_{R}(Q, N)$ is defined as $(\mathrm{P}, \mathrm{M}) \cup_{R}$ $(Q, N)(\mathrm{P}, \mathrm{M}) \cup_{R}(Q, N)=(H, C)$ where $C=M \cup N$

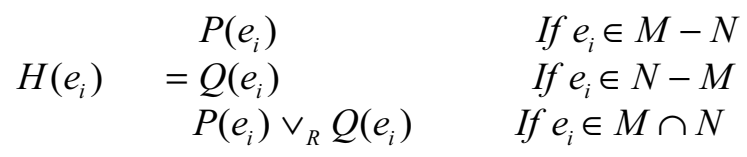

Where $P\left(e_{i}\right) \vee_{R} Q\left(e_{i}\right)$ is defined as $P\left(e_{i}\right) \vee_{R} Q\left(e_{i}\right)=\left\{<\mathrm{x}, \max \left\{\mathrm{A}_{\mathrm{e}_{\mathrm{i}}}(\mathrm{x}), \mathrm{B}_{\mathrm{e}_{\mathrm{i}}}(\mathrm{x})\right\},\left(\lambda_{\mathrm{e}_{\mathrm{i}}} \wedge \mu_{\mathrm{e}_{\mathrm{i}}}\right)(\mathrm{x})>: \mathrm{x} \in \mathrm{X}\right\} \mathrm{e}_{\mathrm{i}} \in$ $\in \mathrm{M} \cap \mathrm{N}\}$ where $P^{T}\left(e_{i}\right) \vee_{R} Q^{T}\left(e_{i}\right)=\left\{<\mathrm{x}, \min \left\{\mathrm{A}_{\mathrm{e}_{\mathrm{i}}}^{\mathrm{T}}(\mathrm{x}), \mathrm{B}_{\mathrm{e}_{\mathrm{i}}}^{\mathrm{T}}(\mathrm{x})\right\},\left(\lambda_{\mathrm{e}_{\mathrm{i}}}^{\mathrm{T}} \wedge \mu_{\mathrm{e}_{\mathrm{i}}}^{\mathrm{T}}\right)\right.$ $\left.(\mathrm{x})>: \mathrm{x} \in \mathrm{X}\} \quad \mathrm{e}_{\mathrm{i}} \in \mathrm{M} \cap \mathrm{N}\right\}$. Given condition is $\left(\lambda_{e_{i}}^{T} \wedge \mu_{e_{i}}^{T}\right)(x) \leq \max \left\{A_{e_{i}}^{-T}\right.$ $\left.(x), B_{e_{i}}^{-T}(x)\right\}$ for all $\mathrm{e}_{\mathrm{i}} \in \mathrm{M}$ and for all $\mathrm{e}_{\mathrm{i}} \in N$ and for all $\mathrm{x} \in \mathrm{X}$. this implies that

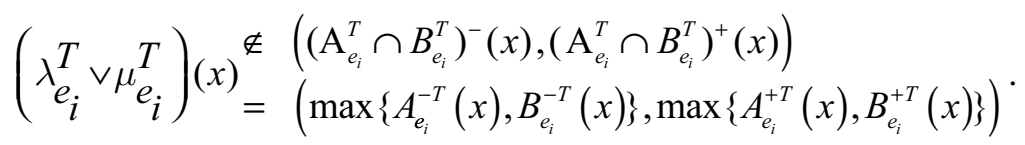

Hence $(\mathrm{P}, \mathrm{M}) \cup_{R}(Q, N)$ is T-ENSCS in $\mathrm{X}$.

Similarly we have the following theorems

\section{Theorem: 3.20}

Let $(\mathrm{P}, \mathrm{M})=\left\{\mathrm{P}\left(\mathrm{e}_{\mathrm{i}}\right)=\left\{<\mathrm{x}, \mathrm{A}_{\mathrm{e}_{\mathrm{i}}}(\mathrm{x}), \lambda_{\mathrm{e}_{\mathrm{i}}}(\mathrm{x})>: \mathrm{x} \in \mathrm{X}\right\} \mathrm{e}_{\mathrm{i}} \in \mathrm{M}\right\}$ and
More on R-Union and R-Intersection of Neutrosophic Soft Cubic Set 
Cruz, RA

Irudayam, FN

$(\mathrm{Q}, \mathrm{N})=\left\{\mathrm{Q}\left(\mathrm{e}_{\mathrm{i}}\right)=\left\{<\mathrm{x}, \mathrm{B}_{\mathrm{e}_{\mathrm{i}}}(\mathrm{x}), \mu_{\mathrm{e}_{\mathrm{i}}}(\mathrm{x})>: \mathrm{x} \in \mathrm{X}\right\} \mathrm{e}_{\mathrm{i}} \in \mathrm{N}\right\}$ be T- INSCSs in X such that $\left(\lambda_{e_{i}}^{I} \wedge \mu_{e_{i}}^{I}\right)(x) \leq \max \left\{A_{e_{i}}^{-I}(x), B_{e_{i}}^{-I}(x)\right\}$ for all $\mathrm{e}_{\mathrm{i}} \in \mathrm{M}$ and for all $\mathrm{e}_{\mathrm{i}} \in N$ and for all $\mathrm{x} \in \mathrm{X}$, then $(\mathrm{P}, \mathrm{M}) \cup_{R}(Q, N)$ is an I-ENSCS in $\mathrm{X}$.

\section{Theorem: 3.21}

Let $(P, M)=\left\{P\left(e_{i}\right)=\left\{<x, A_{e_{i}}(x), \lambda_{e_{i}}(x)>: x \in X\right\} e_{i} \in M\right\}$ and $(\mathrm{Q}, \mathrm{N})=\left\{\mathrm{Q}\left(\mathrm{e}_{\mathrm{i}}\right)=\left\{<\mathrm{x}, \mathrm{B}_{\mathrm{e}_{\mathrm{i}}}(\mathrm{x}), \mu_{\mathrm{e}_{\mathrm{i}}}(\mathrm{x})>: \mathrm{x} \in \mathrm{X}\right\} \mathrm{e}_{\mathrm{i}} \in \mathrm{N}\right\}$ be T- INSCSs in $\mathrm{X}$ such that $\left(\lambda_{e_{i}}^{F} \wedge \mu_{e_{i}}^{F}\right)(x) \leq \max \left\{A_{e_{i}}^{-F}(x), B_{e_{i}}^{-F}(x)\right\}$ for all $\mathrm{e}_{\mathrm{i}} \in M$ and for all $\mathrm{e}_{\mathrm{i}} \in N$ and for all $\mathrm{x} \in \mathrm{X}$, then $(\mathrm{P}, \mathrm{M}) \cup_{R}(Q, N)$ is both an F-ENSCS in $\mathrm{X}$.

\section{Corollary: $\mathbf{3 . 2 2}$}

Let $(\mathrm{P}, \mathrm{M})=\left\{\mathrm{P}\left(\mathrm{e}_{\mathrm{i}}\right)=\left\{<\mathrm{x}, \mathrm{A}_{\mathrm{e}_{\mathrm{i}}}(\mathrm{x}), \lambda_{\mathrm{e}_{\mathrm{i}}}(\mathrm{x})>: \mathrm{x} \in \mathrm{X}\right\} \mathrm{e}_{\mathrm{i}} \in \mathrm{M}\right\}$ and $(\mathrm{Q}, \mathrm{N})=\left\{\mathrm{Q}\left(\mathrm{e}_{\mathrm{i}}\right)=\left\{<\mathrm{x}, \mathrm{B}_{\mathrm{e}_{\mathrm{i}}}(\mathrm{x}), \mu_{\mathrm{e}_{\mathrm{i}}}(\mathrm{x})>: \mathrm{x} \in \mathrm{X}\right\} \mathrm{e}_{\mathrm{i}} \in \mathrm{N}\right\}$ be INSCSs then $(\mathrm{P}, \mathrm{M}) \cup_{R}(Q, N)$ is an ENSCS in $\mathrm{X}$ when the THEOREMS (3.19), (3.20) and (3.21) are valid.

\section{Theorem: $\mathbf{3 . 2 3}$}

Let $(\mathrm{P}, \mathrm{M})=\left\{\mathrm{P}\left(\mathrm{e}_{\mathrm{i}}\right)=\left\{<\mathrm{x}, \mathrm{A}_{\mathrm{e}_{\mathrm{i}}}(\mathrm{x}), \lambda_{\mathrm{e}_{\mathrm{i}}}(\mathrm{x})>: \mathrm{x} \in \mathrm{X}\right\} \mathrm{e}_{\mathrm{i}} \in \mathrm{M}\right\}$ and $(\mathrm{Q}, \mathrm{N})=\left\{\mathrm{Q}\left(\mathrm{e}_{\mathrm{i}}\right)=\left\{<\mathrm{x}, \mathrm{B}_{\mathrm{e}_{\mathrm{i}}}(\mathrm{x}), \mu_{\mathrm{e}_{\mathrm{i}}}(\mathrm{x})>: \mathrm{x} \in \mathrm{X}\right\} \mathrm{e}_{\mathrm{i}} \in \mathrm{N}\right\}$ be T- INSCSs in $\mathrm{X}$ such that $\left(\lambda_{e_{i}}^{T} \vee \mu_{e_{i}}^{T}\right)(x) \geq \max \left\{A_{e_{i}}^{+T}(x), B_{e_{i}}^{+T}(x)\right\}$ for all $\mathrm{e}_{\mathrm{i}} \in M$ and for all $\mathrm{e}_{\mathrm{i}} \in N$ and for all $\mathrm{x} \in \mathrm{X}$, then $(\mathrm{P}, \mathrm{M}) \cap_{R}(Q, N)$ is T-ENSCS in $\mathrm{X}$.

Proof:

Let $(\mathrm{P}, \mathrm{M})=\left\{\mathrm{P}\left(\mathrm{e}_{\mathrm{i}}\right)=\left\{<\mathrm{x}, \mathrm{A}_{\mathrm{e}_{\mathrm{i}}}(\mathrm{x}), \lambda_{\mathrm{e}_{\mathrm{i}}}(\mathrm{x})>: \mathrm{x} \in \mathrm{X}\right\} \mathrm{e}_{\mathrm{i}} \in \mathrm{M}\right\}$ and $(\mathrm{Q}, \mathrm{N})=\left\{\mathrm{Q}\left(\mathrm{e}_{\mathrm{i}}\right)=\left\{<\mathrm{x}, \mathrm{B}_{\mathrm{e}_{\mathrm{i}}}(\mathrm{x}), \mu_{\mathrm{e}_{\mathrm{i}}}(\mathrm{x})>: \mathrm{x} \in \mathrm{X}\right\} \mathrm{e}_{\mathrm{i}} \in \mathrm{N}\right\}$ are T- INSCSs in $\mathrm{X}$.

Thus for all $\mathrm{e}_{\mathrm{i}} \in \mathrm{M}$, we have $A_{e_{i}}^{-T}(x) \leq \lambda_{e_{i}}^{T}(x) \leq A_{e_{i}}^{+T}(x)$ and for all $\mathrm{e}_{\mathrm{i}} \in$ $N B_{e_{i}}^{-T}(x) \leq \mu_{e_{i}}^{T}(x) \leq B_{e_{i}}^{+T}(x)$. Since $(\mathrm{P}, \mathrm{M}) \cap_{R}(Q, N)$ is defined as $(\mathrm{P}, \mathrm{M}) \cap_{R}$ $(Q, N)(P, M) \cap_{R}(Q, N)=(H, C)$ where $C=M \cap N$ and 
$H\left(e_{i}\right)=P\left(e_{i}\right) \wedge_{R} Q\left(e_{i}\right) \quad$ if $e_{i} \in M \cap N$, where $P\left(e_{i}\right) \wedge_{R} Q\left(e_{i}\right)$ is defined as $P\left(e_{i}\right) \wedge_{R} Q\left(e_{i}\right)=\left\{<\mathrm{x}, \min \left\{\mathrm{A}_{\mathrm{e}_{\mathrm{i}}}(\mathrm{x}), \mathrm{B}_{\mathrm{e}_{\mathrm{i}}}(\mathrm{x})\right\},\left(\lambda_{\mathrm{e}_{\mathrm{i}}} \vee \mu_{\mathrm{e}_{\mathrm{i}}}\right)(\mathrm{x})>: \mathrm{x} \in \mathrm{X}\right\} \mathrm{e}_{\mathrm{i}} \in$ $\mathrm{M} \cap \mathrm{N}\}$ where $P^{T}\left(e_{i}\right) \wedge_{R} Q^{T}\left(e_{i}\right)=$ $\left.\left\{<\mathrm{x}, \min \left\{\mathrm{A}_{\mathrm{e}_{\mathrm{i}}}^{\mathrm{T}}(\mathrm{x}), \mathrm{B}_{\mathrm{e}_{\mathrm{i}}}^{\mathrm{T}}(\mathrm{x})\right\},\left(\lambda_{\mathrm{e}_{\mathrm{i}}}^{\mathrm{T}} \vee \mu_{\mathrm{e}_{\mathrm{i}}}^{\mathrm{T}}\right)(\mathrm{x})>: \mathrm{x} \in \mathrm{X}\right\} \quad \mathrm{e}_{\mathrm{i}} \in \mathrm{M} \cap \mathrm{N}\right\}$.

Given condition is $\left(\lambda_{e_{i}}^{T} \vee \mu_{e_{i}}^{T}\right)(x) \geq \min \left\{A_{e_{i}}^{+T}(x), B_{e_{i}}^{+T}(x)\right\}$

for all $\mathrm{e}_{\mathrm{i}} \in \mathrm{M}$ and for all $\mathrm{e}_{\mathrm{i}} \in N$ and for all $\mathrm{x} \in \mathrm{X}$. this implies that

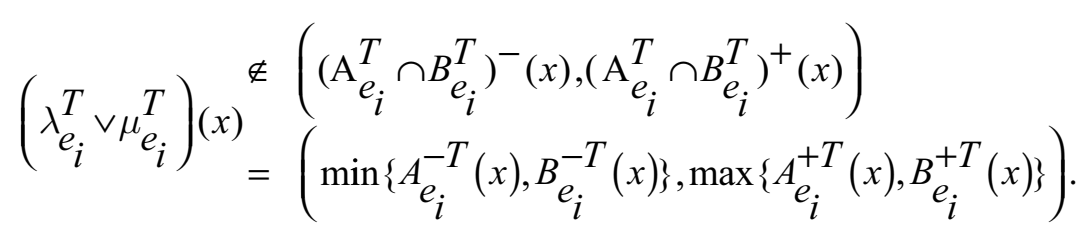

Hence $(\mathrm{P}, \mathrm{M}) \cap_{R}(Q, N)$ is both an T-ENSCS in $\mathrm{X}$.

\section{Theorem 3.24}

Let $(\mathrm{P}, \mathrm{M})=\left\{\mathrm{P}\left(\mathrm{e}_{\mathrm{i}}\right)=\left\{<\mathrm{x}, \mathrm{A}_{\mathrm{e}_{\mathrm{i}}}(\mathrm{x}), \lambda_{\mathrm{e}_{\mathrm{i}}}(\mathrm{x})>: \mathrm{x} \in \mathrm{X}\right\} \mathrm{e}_{\mathrm{i}} \in \mathrm{M}\right\}$ and $(\mathrm{Q}, \mathrm{N})=\left\{\mathrm{Q}\left(\mathrm{e}_{\mathrm{i}}\right)=\left\{<\mathrm{x}, \mathrm{B}_{\mathrm{e}_{\mathrm{i}}}(\mathrm{x}), \mu_{\mathrm{e}_{\mathrm{i}}}(\mathrm{x})>: \mathrm{x} \in \mathrm{X}\right\} \mathrm{e}_{\mathrm{i}} \in \mathrm{N}\right\}$ be I- INSCSs in $\mathrm{X}$ such that $\left(\lambda_{e_{i}}^{I} \vee \mu_{e_{i}}^{I}\right)(x) \geq \max \left\{A_{e_{i}}^{+I}(x), B_{e_{i}}^{+I}(x)\right\}$ for all $\mathrm{e}_{\mathrm{i}} \in \mathrm{M}$ and for all $\mathrm{e}_{\mathrm{i}} \in N$ and for all $\mathrm{x} \in \mathrm{X}$.then $(\mathrm{P}, \mathrm{M}) \cap_{R}(Q, N)$ is an I-ENSCS in $\mathrm{X}$.

\section{Theorem 3.25}

Let $(\mathrm{P}, \mathrm{M})=\left\{\mathrm{P}\left(\mathrm{e}_{\mathrm{i}}\right)=\left\{<\mathrm{x}, \mathrm{A}_{\mathrm{e}_{\mathrm{i}}}(\mathrm{x}), \lambda_{\mathrm{e}_{\mathrm{i}}}(\mathrm{x})>: \mathrm{x} \in \mathrm{X}\right\} \mathrm{e}_{\mathrm{i}} \in \mathrm{M}\right\}$ and $(\mathrm{Q}, \mathrm{N})=\left\{\mathrm{Q}\left(\mathrm{e}_{\mathrm{i}}\right)=\left\{<\mathrm{x}, \mathrm{B}_{\mathrm{e}_{\mathrm{i}}}(\mathrm{x}), \mu_{\mathrm{e}_{\mathrm{i}}}(\mathrm{x})>: \mathrm{x} \in \mathrm{X}\right\} \mathrm{e}_{\mathrm{i}} \in \mathrm{N}\right\}$ be F- INSCSs in X such that $\left(\lambda_{e_{i}}^{F} \vee \mu_{e_{i}}^{F}\right)(x) \geq \max \left\{A_{e_{i}}^{+F}(x), B_{e_{i}}^{+F}(x)\right\}$ for all $\mathrm{e}_{\mathrm{i}} \in \mathrm{M}$ and for all $\mathrm{e}_{\mathrm{i}} \in N$ and for all $\mathrm{x} \in \mathrm{X}$. then $(\mathrm{P}, \mathrm{M}) \cap_{R}(Q, N)$ is F-ENSCS in $\mathrm{X}$.

\section{Corollary: $\mathbf{3 . 2 6}$}

Let $(\mathrm{P}, \mathrm{M})=\left\{\mathrm{P}\left(\mathrm{e}_{\mathrm{i}}\right)=\left\{<\mathrm{x}, \mathrm{A}_{\mathrm{e}_{\mathrm{i}}}(\mathrm{x}), \lambda_{\mathrm{e}_{\mathrm{i}}}(\mathrm{x})>: \mathrm{x} \in \mathrm{X}\right\} \mathrm{e}_{\mathrm{i}} \in \mathrm{M}\right\}$ and 
Cruz, RA Irudayam, FN

$(\mathrm{Q}, \mathrm{N})=\left\{\mathrm{Q}\left(\mathrm{e}_{\mathrm{i}}\right)=\left\{<\mathrm{x}, \mathrm{B}_{\mathrm{e}_{\mathrm{i}}}(\mathrm{x}), \mu_{\mathrm{e}_{\mathrm{i}}}(\mathrm{x})>: \mathrm{x} \in \mathrm{X}\right\} \mathrm{e}_{\mathrm{i}} \in \mathrm{N}\right\}$ be INSCSs then $(\mathrm{P}, \mathrm{M}) \cap_{R}(Q, N)$ is both an ENSCS in $\mathrm{X}$ when the Theorems (3.23), (3.24) and (3.25) are valid.

\section{REFERENCES}

[1] R. Anitha Cruz and F. Nirmala Irudayam, Neutrosophic Soft Cubic Set, International Journal of Mathematics Trends andTechnology, 46(2), 88-94(2017).

[2] R. Anitha Cruz and F. Nirmala Irudayam, More on P-union and P- intersection of Neutrosophic Soft Cubic Set, Neutrosophic Sets and Systems (communicated).

[3] Atanassov, K. (1986). "Intuitionistic fuzzy sets". Fuzzy Sets and Systems 20, 87-96.

[4] D. Molodtsov, Soft Set Theory-First Results, Computers and Mathematics with Application, 37, 19-31 (1999).

[5] Pabita Kumar Majii, "Neutrosophic soft set" Annals of Fuzzy Mathematics and Informatics 5, 157-168 (2013).

[6] P.K. Majii , R. Biswas and A.R. Roy, "Fuzzy soft sets", Journal of Fuzzy Mathematics, Vol 9,no. 3, 589-602, (2001).

[7] P.K. Majii, R. Biswas and A.R .Roy, "Intuitionistic Fuzzy soft sets", The journal of fuzzy Mathematics, Vol 9, (3) 677-692 (2001).

[8] L. J. Kohout, W. Bandler,. (1996) Fuzzy interval inference utilizing the checklist paradigm and BK relational products, in: R.B. Kearfort et al.(Eds.), Applications of Interval Computations, (pp. 291-335), Kluwer, Dordrecht.

[9] F. Smarandache. (1999) A Unifying Field in Logics. Neutrosophy: Neutrosophic Probability, Set and Logic, Rehoboth: American Research Press.

[10] F. Smarandache, Neutrosophic set, a generalization of the Intuitionistic fuzzy sets, Inter. J. Pu Bre Appl. Math. 24, 287-297(2005).

[11] F. Smarandache, Neutrosophy and Neutrosophic Logic, (2002) First International Conference on Neutrosophy, Neutrosophy Logic, Set, Probability and Statistics University of New Mexico, Gallup, NM 87301, USA

[12] I. B. Turksen, Interval-valued fuzzy sets and compensatory AND, Fuzzy Sets and Systems 51, 295-307(1992).

[13] Turksen, "Interval valued fuzzy sets based on normal forms". Fuzzy Sets and Systems, 20, 191-210, (1968).

[14] I. B. Turksen, Interval-valued strict preference with Zadeh triples, Fuzzy Sets and Systems 78, 183-195 (1996).

[15] H. Wang, F. Smarandache, Y.Q. Zhang and R. Sunderraman, (2005) Interval Neutrosophic Sets and logic: Theory and Applications in Computing, Hexis; Neutrosophic book series, No.5. 
[16] Y.B. Jun, C.S. Kim and K.O. Yang, Cubic sets, Annals of Fuzzy Mathematics and Informatics 4(3), 83-98 (2012),

[17] Young Bae Jun, Florentin Smarandache, and Chang Su Kim, R-intersections and R-unions of neutrosophic cubic sets, IEEE International Conference on Fuzzy Systems, 2441-2443 (2016) .

[18] Jun YB, Smarandache F, Kim CS (2017) Neutrosophic cubic sets. New Mathematics and Natural Computation 13, 41-54

[19] L.A. Zadeh, Fuzzy sets, Inform Control 8, 338-353 (1965).
More on R-Union and R-Intersection of Neutrosophic Soft Cubic Set 